\title{
A LIAPUNOV-SCHMIDT REDUCTION FOR TIME-PERIODIC SOLUTIONS OF THE COMPRESSIBLE EULER EQUATIONS*
}

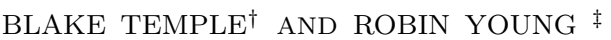

\begin{abstract}
Following the authors' earlier work in [8, 9], we show that the nonlinear eigenvalue problem introduced in [9] can be recast in the language of bifurcation theory as a perturbation of a linearized eigenvalue problem. Solutions of this nonlinear eigenvalue problem correspond to time periodic solutions of the compressible Euler equations that exhibit the simplest possible periodic structure identified in [8]. By a Liapunov-Schmidt reduction we establish and refine the statement of a new infinite dimensional KAM type small divisor problem in bifurcation theory, whose solution will imply the existence of exact time-periodic solutions of the compressible Euler equations. We then show that solutions exist to within an arbitrarily high Fourier mode cutoff. The results introduce a new small divisor problem of quasilinear type, and lend further strong support for the claim that the time-periodic wave pattern described at the linearized level in [9], is physically realized in nearby exact solutions of the fully nonlinear compressible Euler equations.
\end{abstract}

Key words. Compressible Euler, periodic solutions, conservation laws.

AMS subject classifications. 35L65, 76N10.

1. Introduction. In [8] the authors derived a simplest possible periodic wave structure $^{1}$ consistent with time-periodic sound wave propagation in the $3 \times 3$ nonlinear compressible Euler equations. This wave structure requires at least three coupled nonlinear equations to support it. The wave pattern was derived by combinatorial considerations based on a classification of compressive and rarefactive wave interactions at entropy jumps, using the starting principle that shock free periodic or quasi-periodic solutions of compressible Euler should balance compression and rarefaction along every characteristic (sound wave). This starting work was followed by the authors' work in [9] in which we construct exact linearized solutions of Euler that exhibit the wave structure identified in [8] for the nonlinear problem. For this we derived a nonlinear eigenvalue problem of the form

$$
\mathcal{N}[V]=V
$$

whose solutions correspond to nonlinear periodic solutions of the compressible Euler equations having the simplest structure identified in [8]. The nonlinear operator $\mathcal{N}$ consists of compositions of nonlinear spatial evolution, entropy jumps and shift operators, starting from time-periodic Cauchy data posed at $x=0$. These are noncommuting operators, constructed to reproduce the periodic structure identified in [8]. Trivial solutions of (1) consist of piecewise constant states separated by entropy jumps (contact discontinuities). Linearizing around such solutions, we obtained a linearized

\footnotetext{
*Received April 11, 2010; accepted for publication September 17, 2010.

$\dagger$ Department of Mathematics, University of California, Davis, CA 95616, USA (temple@ math.ucdavis.edu). Supported in part by NSF Applied Mathematics Grant Number DMS-040-6096.

${ }^{\ddagger}$ Department of Mathematics and Statistics, University of Massachusetts, Amherst, MA 01003, USA (young@math.umass.edu). Supported in part by NSF Applied Mathematics Grant Number DMS-050-7884.

${ }^{1}$ We say that a periodic or quasi-periodic wave structure is possible, at a formal level, if each characteristic (sound wave), traverses both regions of compression and rarefaction, and that these are formally in balance. Whether such a possible formal wave structure actually exists in a true periodic solution of Euler is then a deep mathematical question as to whether the data can be tuned to bring compression and rarefaction precisely into balance, so that shock wave formation is prevented.
} 
eigenvalue problem

$$
\mathcal{M}[V]=V
$$

whose solutions we expect will perturb to solutions of the nonlinear problem because they encode the structure identified in [8]. The linearized operator $\mathcal{M}$ is nonsymmetric, and consists of the composition of five elementary linear operators that do not commute: a linear spatial evolution at the first entropy level, followed by an entropy jump, followed by linear spatial evolution at the second entropy level, followed by the inverse entropy jump, followed by a half period shift. The combination of shifts and jumps ensures the mixing of compression and rarefaction along characteristics under nonlinear perturbation, and highly restricts the kernel of the linearized operator. To construct solutions in [9] we derived a condition, relating the magnitude of the entropy jump to the two spatial periods, that guarantees the existence of a solution to the linear eigenvalue problem in the Fourier 1-mode. The periodic linearized solutions of Euler were then obtained by deriving closed form expressions for the resulting 1-mode solutions of the linearized system of PDEs.

These linearized solutions display, in closed form expression, the propagation properties of nearby nonlinear sound waves that formally balance compression and rarefaction along characteristics. In this sense, the solutions exhibit the simplest possible mechanism for dissipation free transmission of sound waves in the nonlinear problem. In [9] we went on to analyze the spectrum of the linearized operator that expresses the eigenvalue problem corresponding to periodicity. We proved that the linearized periodic solutions correspond to eigenvectors in the 1-mode kernel of the associated linearized operators, and that for almost every choice of periods, the linearized operator is non-resonant in the sense that it is invertible on the complement of the 1-mode kernel, c.f. [2]. Interestingly, in [9], it emerged that the linearized operator is non-resonant only in the case when the sound speeds are incommensurate with the periods. In the special case of symmetric periods (when the non-dimensionalized spatial widths of constant entropy are equal), we showed that the eigenvalues are bounded away from zero by algebraic rates in the Fourier modes.

In this paper we show that the nonlinear eigenvalue problem (1) can be recast as a perturbation from the linear eigenvalue problem (2). We then show that the resulting bifurcation problem is amenable to a Liapunov-Schmidt reduction, and perform the reduction in the case of arbitrary non-resonant periods. The Liapunov-Schmidt method involves two applications of the implicit function theorem, one to solve the auxiliary equation associated with the nonsingular operator defined on the orthogonal complement of the kernel, and one to solve the bifurcation equation associated with the kernel of (2); a solution of the second equation hinges on existence for the first, [4]. In this paper we prove that the Liapunov-Schmidt method is valid, subject to the existence of solutions to the auxiliary equation. This reduces the problem of existence of time-periodic solutions of the compressible Euler equations to a KAM-type implicit function theorem for an inverible linear operator with small divisors. As a corollary, we prove that periodic solutions exist subject to an arbitrarily high Fourier mode cutoff.

The search for a complete proof of existence of solutions of the KAM type implicit function theorem posed by the auxiliary equation is the topic of the authors' ongoing research program. This involves an infinite dimensional space and a nonlinear nonlocal differential operator $\mathcal{N}$ of quasilinear type, and so, as far as we know, is beyond the direct application of known results. Similar problems have been resolved in the 
semilinear setting with the same estimates on the small divisors, and with weaker results on the structure of the kernel, c.f $[2,1]$. The new feature of our problem is that the quasilinear problem reduces to an ODE in a Hilbert space, but there is no apparent basis in which the problem becomes an infinite algebraic system as in $[2,1]$.

The Liapunov-Schmidt reduction that applies in the infinite dimensional case also applies when we impose a Fourier cutoff of the nonlinear problem up to arbitrarily high Fourier modes, a non-trivial problem in its own right. In the case of an $N$-Fourier mode cutoff, $N$ arbitrarily large, we prove that the resulting auxiliary equation has a unique solution ${ }^{2}$. This shows that periodic solutions with the wave structure identified in $[8,9]$ exist up to an arbitrary Fourier cutoff, and lends further strong support to the claim that the wave pattern described in [8] is physically realized.

The paper is laid out as follows: in Section 2, we restate the problem of existence as an eigenvalue problem, and recall the the linearized solutions from [9]. In Section 3, we set notation and give the Liapunov-Schmidt reduction of the perturbation problem. In Section 4 we study the bifurcation equation, and in Section 5, we solve the auxiliary equation for the $N$-th mode Fourier cutoff problem.

\section{Background and previous results.}

2.1. The nonlinear equations. We are looking for periodic solutions of the compressible Euler equations, which describe the evolution of a perfect fluid in the absence of dissipative effects. For one-dimensional flow, this is the $3 \times 3$ system

$$
\begin{aligned}
\rho_{t}+(\rho u)_{x} & =0, \\
(\rho u)_{t}+\left(\rho u^{2}+p\right)_{x} & =0, \\
E_{t}+[(E+p) u]_{x} & =0,
\end{aligned}
$$

representing conservation of mass, momentum and energy, respectively. Here $x$ is the Eulerian spatial coordinate and the state variables are density $\rho$, pressure $p$, velocity $u$ and energy density $E=\frac{1}{2} \rho u^{2}+\rho \varepsilon$, where $\varepsilon$ is the specific internal energy. To close the system, an equation of state is given which relates the thermodynamic variables $p, \varepsilon$ and $\rho$. We consider a polytropic gamma-law gas, described by

$$
\varepsilon=c_{\tau} \tau^{-(\gamma-1)} e^{S / c_{\tau}} \text { and } p=\frac{c_{\tau}}{\gamma-1} \tau^{-\gamma} e^{S / c_{\tau}},
$$

where $\tau=1 / \rho$ is the specific volume, $S$ is the specific entropy, $\gamma>1$ is the adiabatic gas constant, and $c_{\tau}$ the specific heat [7]. On regions where the solution is smooth, the energy equation is equivalent to the adiabatic constraint or entropy equation

$$
(\rho S)_{t}+(\rho S u)_{x}=0,
$$

which states that entropy is transported with the fluid [7].

In a Lagrangian frame, co-moving with the fluid, the equations are

$$
\begin{aligned}
\tau_{t}-u_{x} & =0, \\
u_{t}+p_{x} & =0, \\
E_{t}^{*}+(u p)_{x} & =0,
\end{aligned}
$$

\footnotetext{
${ }^{2}$ Interestingly, under the Fourier cutoff assumption, we obtain periodic solutions for non-resonant $\Theta$ as well as nearby resonant $\Theta$, because resonant values of $\Theta$ lie arbitrarily close to non-resonant values, and so are captured under perturbation. This then raises the interesting question as to whether the small divisors are just an anomaly of the linearization process that go away at the next order of approximation of the nonlinear problem, or whether they are essential for perturbation. The authors intend to address this problem in a future paper.
} 
where now $x$ is the Lagrangian material coordinate for the fluid, given by replacing

$$
\int \rho d x \rightarrow x
$$

and $E^{*}=\frac{1}{2} u^{2}+\varepsilon(\tau, S)$. In this Lagrangian frame, the adiabatic constraint (5) takes on the particularly simple form

$$
S_{t}=0
$$

which can be used in place of the energy equation on smooth regions [7].

We recall from $[8,9]$ the convenient change of variables

$$
m=e^{S / 2 c_{\tau}} \quad \text { and } \quad z=K_{z} \tau^{-\frac{\gamma-1}{2}},
$$

so that (4) becomes

$$
\varepsilon=K_{\varepsilon} m^{2} z^{2} \quad \text { and } \quad p=K_{p} m^{2} z^{\frac{2 \gamma}{\gamma-1}},
$$

where $K$.'s are appropriately given constants. In these variables, on regions of smooth solution, our equations (6), (7) reduce to the quasilinear system

$$
\begin{aligned}
z_{t}+\frac{c}{m} u_{x} & =0 \\
u_{t}+m c z_{x}+2 \frac{p}{m} m_{x} & =0 \\
m_{t} & =0
\end{aligned}
$$

where we have used entropy (7) in place of the energy; here $c$ is the Lagrangian sound speed, defined by

$$
c(\tau, S)=\sqrt{-p_{\tau}(\tau, S)}
$$

which becomes

$$
c(m, z)=K_{c} m z^{d} \quad \text { with } \quad d \equiv \frac{\gamma+1}{\gamma-1} .
$$

We are interested in the particular class of piecewise smooth solutions with piecewise constant entropy; for this class, the $3 \times 3$ system (10) reduces to the $2 \times 2$ quasilinear system

$$
\begin{aligned}
z_{t}+\frac{c}{m} u_{x} & =0, \\
u_{t}+m c z_{x} & =0,
\end{aligned}
$$

which is just the $p$-system of isentropic gas dynamics, with $z$ as the thermodynamic coordinate $[7,10]$. We will thus solve the simpler $2 \times 2$ system (12) in different regions, with different entropy levels corresponding to different constants $m$ in the constitutive relation (11).

The class of solutions we consider does have discontinuities, so we must treat these correctly, namely by using the Rankine-Hugoniot jump conditions. We apply these to the fully nonlinear system (6), to get

$$
\begin{aligned}
{[u] } & =s[-\tau] \\
{[p] } & =s[u] \\
{[u p] } & =s\left[\frac{1}{2} u^{2}+\varepsilon\right],
\end{aligned}
$$


where $s$ is the speed of propagation of the discontinuity and [.] is the jump. For our special class of solutions, the only discontinuities are stationary entropy jumps with $s=0$, so the jump conditions reduce to $[u]=[p]=0$, or, by $(9)$,

$$
u_{L}=u_{R} \quad \text { and } \quad m_{L}^{2} z_{L}^{\frac{2 \gamma}{\gamma-1}}=m_{R}^{2} z_{R}^{\frac{2 \gamma}{\gamma-1}} .
$$

We are interested in fixed stationary entropy jumps, so that $m_{L}$ and $m_{R}$ are fixed, and we get a diagonal linear operator $\left(u_{L}, z_{L}\right) \rightarrow\left(u_{R}, z_{R}\right)$.

2.2. The nonlinear eigenvalue problem. In our previous papers $[8,9]$, we described the physical mechanism by which periodic solutions can be supported. In short, we impose the structure of a fixed piecewise constant entropy profile, and superpose on this a periodic structure of nonlinear simple waves. This periodic structure must be very carefully chosen so that across entropy jumps, which are stationary contact discontinuities, some parts of the nonlinear simple waves change type from rarefaction to compression (and vice versa); moreover, this must be done consistently. This change of character of the waves is the fundamental nonlinear effect that balances the tendency of nonlinear waves to compress and form shocks, which are incompatible with periodic evolution.

Because we restrict to the class of solutions which are piecewise smooth with piecewise constant entropy profiles and no other discontiunities, the nonlinear evolution for this class is given by the $2 \times 2$ system (12) together with (14) at the jumps. We now reformulate the problem of existence of a periodic solution as a nonlinear eigenvalue (fixed point) problem.

In $[8,9]$, we considered the simplest case which supports periodicity, namely two entropy levels $\bar{m}>\underline{m}$ of fixed width $\bar{x}$ and $\underline{x}$, respectively, continued periodically. We then solve (12) on top of this entropy structure, denoting the corresponding states by $\bar{U}=(\bar{z}, \bar{u})$ and $\underline{U}=(\underline{z}, \underline{u})$, respectively. Thus $\bar{U}(x, t) \equiv(\bar{z}(x, t), \bar{u}(x, t))$ solves

$$
\begin{aligned}
z_{t}+\frac{\bar{c}(z)}{\bar{m}} u_{x} & =0, \\
u_{t}+\bar{m} \bar{c}(z) z_{x} & =0,
\end{aligned}
$$

in the interval $0<x<\bar{x}$, and $\underline{U}(x, t) \equiv(\underline{z}(x, t), \underline{u}(x, t))$ solves

$$
\begin{aligned}
z_{t}+\frac{\underline{c}(z)}{\underline{m}} u_{x} & =0, \\
u_{t}+\underline{m} \underline{c}(z) z_{x} & =0,
\end{aligned}
$$

in $\bar{x}<x<\bar{x}+\underline{x}$, respectively. Such a solution defined on the bounded region (or tile of the plane)

$$
0<x<\bar{x}+\underline{x}, \quad 0 \leq t<2 \pi,
$$

generates a space-periodic solution of Euler provided appropriate "boundary conditions" are met. Since we are interested in solving the full Euler equations, our matching condition at each entropy jump is exactly (14), and, in order to appropriately mix the simple waves, the region tiles the $(x, t)$-plane with a half-period time shift.

To be more precise, we make the solution $2 \pi$-periodic in time $t$, and we regard $x$ as the evolution variable in (15), (16). That is, we consider as data the function

$$
\dot{U}(t) \equiv U(0+, t)=\bar{U}(0, t),
$$


and evolve this data to

$$
\check{U}(t) \equiv U(\bar{x}-, t)=\bar{U}(\bar{x}, t),
$$

where $\bar{U}(x, t)$ satisfies $(15)$ for $0<x<\bar{x}$. We then jump this by the Rankine-Hugoniot condition (14), to

$$
\tilde{U}(t) \equiv U(\bar{x}+, t)=\underline{U}(\bar{x}, t),
$$

and evolve $\underline{U}(x, t)$ by $(16)$ to

$$
\hat{U}(t) \equiv U(\bar{x}+\underline{x}-, t)=\underline{U}(\bar{x}+\underline{x}, t),
$$

for $\bar{x}<x<\bar{x}+\underline{x}$. The solution thus defined generates a periodic tile of the $(x, t)$-plane if $\hat{U}$ and some time-shift of $\dot{U}$ are related by the jump condition (14). For convenience, we impose maximal symmetry by fixing the time-shift as one-half period, $\pi$.

We describe this succinctly as follows: define the nonlinear evolution operators $\overline{\mathcal{E}}$ and $\underline{\mathcal{E}}$ by evolution (in the spatial variable $x$ ) using system (15), (16) of $U(t)$ through $\bar{x}$ and $\underline{x}$, respectively. In this notation,

$$
\check{U}=\overline{\mathcal{E}} \dot{U} \quad \text { and } \quad \hat{U}=\underline{\mathcal{E}} \tilde{U} .
$$

Next, define the jump operator $\mathcal{J}$ which maps $U_{L}(t)$ to $U_{R}(t)$ by the Hugoniot conditions (14) with $m_{L}=\bar{m}$ and $m_{R}=\underline{m}$; thus $\mathcal{J}$ is just the diagonal linear operator

$$
\mathcal{J}=\left(\begin{array}{cc}
J_{1} & 0 \\
0 & 1
\end{array}\right), \quad \text { where } \quad J_{1} \equiv\left(\frac{\bar{m}}{\underline{m}}\right)^{\frac{\gamma-1}{\gamma}} \text {. }
$$

Since (14) is symmetric, the jump operator at the other entropy jump is just $\mathcal{J}^{-1}$, so we have

$$
\tilde{U}=\mathcal{J} \check{U}, \quad \text { and we set } \quad U^{*} \equiv \mathcal{J}^{-1} \hat{U}
$$

so that $U^{*}$ is at the same entropy level as our data $\dot{U}$. Finally, we define the shift operator

$$
\mathcal{S} U(t) \equiv U(t-\pi)=U(t+\pi)
$$

where we have used a half-period shift to ensure maximal symmetry. Our "periodic boundary condition" above then becomes $\dot{U}=\mathcal{S} U^{*}$, and combining these relations, we see that the existence of a periodic solution having this structure is equivalent to the fixed point (eigenvalue) problem

$$
\mathcal{S} \cdot \mathcal{J}^{-1} \cdot \underline{\mathcal{E}} \cdot \mathcal{J} \cdot \overline{\mathcal{E}} \dot{U}=\dot{U}
$$

having a sufficiently smooth solution. Here the data is the $2 \pi$-periodic function $\dot{U}(t)$, and we regard each operator as a map from the space of smooth $2 \pi$-periodic functions to itself.

2.3. Non-dimensionalization. Our calculations are simplified by a nondimensionalization which has the effect of removing all explicit references to the entropy $m$. Since we will be linearizing the eigenvalue problem (17), it is natural to identify "base states" $\bar{z}_{0}$ and $\underline{z}_{0}$, which will satisfy the jump condition, so we set 
$J_{1} \bar{z}_{0}=\underline{z}_{0}, \bar{u}_{0}=\underline{u}_{0}$. Letting $z, u$ refer to either $\bar{z}, \bar{u}$ or $\underline{z}, \underline{u}$ in the appropriate strip, and factoring out the base states, (12) can be written

$$
\begin{aligned}
& \left(\frac{z}{z_{0}}\right)_{t}+\frac{c(z)}{c_{0}}\left(\frac{u-u_{0}}{m_{0} z_{0}}\right)_{y}=0 \\
& \left(\frac{u-u_{0}}{m_{0} z_{0}}\right)_{t}+\frac{c(z)}{c_{0}}\left(\frac{z}{z_{0}}\right)_{y}=0
\end{aligned}
$$

where we have set $c_{0}=c_{0}\left(m_{0}, z_{0}\right)$ in (11), and we have rescaled the spatial variable by

$$
\frac{d y}{d x}=\frac{1}{c_{0}}, \quad \text { so that } \quad y-y_{0}=\frac{x-x_{0}}{c_{0}} .
$$

We thus define the dimensionless variables

$$
w=\frac{z}{z_{0}} \quad \text { and } \quad v=\frac{u-u_{0}}{m_{0} z_{0}},
$$

and we make the spatial evolution explicit by writing the system as

$$
\begin{aligned}
& w_{y}+\sigma(w) v_{t}=0, \\
& v_{y}+\sigma(w) w_{t}=0,
\end{aligned}
$$

where we have used (11) to define

$$
\sigma=\frac{c\left(z_{0}\right)}{c(z)}=\frac{z_{0}^{d}}{z^{d}}=w^{-d} \equiv \sigma(w) .
$$

Note that in these dimensionless variables the base state is $\left(w_{0}, v_{0}\right)=(1,0)$, and $\sigma\left(w_{0}\right)=1$. Using (19) in (14), we find that, in dimensionless variables, the jump conditions become

$$
\bar{w}=\underline{w} \quad \text { and } \quad \bar{m}^{\frac{d-1}{d+1}} \bar{v}=\underline{m}^{\frac{d-1}{d+1}} \underline{v}
$$

and in particular, $w$ and the speed $\sigma(w)$ are continuous across the jump.

According to (18), the new widths of the entropy levels will be

$$
\bar{\theta} \equiv \frac{\bar{x}}{c\left(\bar{z}_{0}\right)} \quad \text { and } \quad \underline{\theta} \equiv \frac{\underline{x}}{c\left(\underline{z}_{0}\right)}
$$

and we note that entropy $m$ does not appear explicitly in (19): that is, there is only one evolution operator in these variables. Denoting the dimensionless variables by $V=(w, v)$, we define the evolution operator $\mathcal{E}(\theta)$ using system (20) through a $y$-interval of length $\theta$, so that

$$
\mathcal{E}(\theta) V(0, \cdot)=V(\theta, \cdot),
$$

where $V(y, t)$ solves $(20)$ with Cauchy data $V(0, \cdot)$. Again denoting the jump operator by $\mathcal{J}$, we have

$$
\mathcal{J}=\left(\begin{array}{ll}
1 & 0 \\
0 & J
\end{array}\right), \quad \text { where } \quad J \equiv\left(\frac{\bar{m}}{\underline{m}}\right)^{\frac{1}{\gamma}}=\left(\frac{\bar{m}}{\underline{m}}\right)^{\frac{d-1}{d+1}}
$$


this ratio $J$ is the only place that entropy features in the dimensionless problem. The following restatement of the problem is proved in [9]:

THEOREM 1. For fixed parameters $\bar{\theta}, \underline{\theta}$ and $J$, define the nonlinear operator $\mathcal{N} \equiv \mathcal{N}(\bar{\theta}, \underline{\theta}, J)$ by

$$
\mathcal{N} \equiv \mathcal{S} \cdot \mathcal{J}^{-1} \cdot \mathcal{E}(\underline{\theta}) \cdot \mathcal{J} \cdot \mathcal{E}(\bar{\theta})
$$

and let $V(t)=(w(t), v(t))$ denote any smooth solution of

$$
\mathcal{N} V(\cdot)=V(\cdot)
$$

with averages

$$
w_{0} \equiv \frac{1}{2 \pi} \int_{0}^{2 \pi} w(t) d t=1, \quad \text { and } \quad v_{0} \equiv \frac{1}{2 \pi} \int_{0}^{2 \pi} v(t) d t=0,
$$

respectively. Then given any base state $U_{0}=\left(\bar{z}_{0}, \bar{u}_{0}\right)$ and entropy state $\bar{m}$, there is a periodic solution $U(x, t)=(z(x, t), u(x, t))$ of (10), determined uniquely by $V(t)$, with corresponding average values

$$
\frac{1}{2 \pi} \int_{0}^{2 \pi} z(0, t) d t=\bar{z}_{0}, \quad \text { and } \quad \frac{1}{2 \pi} \int_{0}^{2 \pi} u(0, t) d t=\bar{u}_{0} .
$$

Note that the second base state $\left(\underline{z}_{0}, \underline{m}, \underline{u}_{0}\right)$ is determined by the given state $\left(\bar{z}_{0}, \bar{m}, \bar{u}_{0}\right)$ and jump $J$, and the widths $\bar{x}$ and $\underline{x}$ of the entropy levels are determined from $(\bar{\theta}, \underline{\theta})$ by $(23)$.

2.4. Linearization. Having formulated the nonlinear eigenvalue problem, we linearize the nonlinear operator around the base state $(1,0)$. The fully nonlinear operator $\mathcal{N}$, given by (26), is a composition of nonlinear evolution $\mathcal{E}$ and linear operators; moreover, all operators fix the state $(1,0)$. It follows that the linearization of $\mathcal{N}$ is obtained by linearizing the evolution operator around $(1,0)$. This in turn is easily found: since $\sigma(1)=1$, the linearization of (20) is simply the system

$$
\begin{aligned}
& w_{y}+v_{t}=0, \\
& v_{y}+w_{t}=0,
\end{aligned}
$$

which is just the linear wave equation.

As in (24), we define the linearized evolution operator $\mathcal{L}(\theta)$ by

$$
\mathcal{L}(\theta) V(0, \cdot)=V(\theta, \cdot),
$$

where now $V(y, t)$ solves $(30)$ with Cauchy data $V(0, \cdot)$. Also, define the linear operator $\mathcal{M} \equiv \mathcal{M}(\bar{\theta}, \underline{\theta}, J)$ by

$$
\mathcal{M} \equiv \mathcal{S} \cdot \mathcal{J}^{-1} \cdot \mathcal{L}(\underline{\theta}) \cdot \mathcal{J} \cdot \mathcal{L}(\bar{\theta})
$$

Then $\mathcal{M}$ is the linearization of $\mathcal{N}$ about the base state $(1,0)$, and the linearized eigenvalue problem is

$$
\mathcal{M} V(\cdot)=V(\cdot)
$$

where $V(\cdot)$ is a smooth $2 \pi$-periodic function. 
We remark that although the base state $(1,0)$ is a trivial solution of the $2 \times 2$ evolution and eigenvalue problems, when regarded as a solution of the $3 \times 3$ Euler equations, it is not a trivial solution. That is, although there are no genuinely nonlinear waves propagating, the stationary entropy jumps are nontrivial waves. Indeed, the nonlinear effects of these jumps are seen in the (linearized) $2 \times 2$ eigenvalue problem. In other words, the effects of the jumps are manifested as non-commuting linear operators in (32). In particular, even though linearized waves are not rarefactive or compressive, we can still identify the change of type of simple waves identified in [8] at the linearized level.

Our eigenvalue problems (27), (32) are invariant under an arbitrary phase shift $t \rightarrow t+t_{0}$; a natural way to remove this invariance is to observe that the systems (20), (30) are invariant under the mapping

$$
w(y, t) \rightarrow w(y,-t) \text { and } v(y, t) \rightarrow-v(y,-t)
$$

and thus preserve the properties that $w, v$ be even/odd, respectively. That is, if the

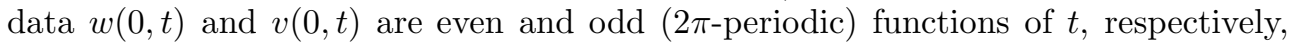
then so are the solutions $w(y, t)$ and $v(y, t)$ for $y>0$; clearly this property is also preserved by the jumps and shift. We emphasize that this fact holds for both the linear and nonlinear problems.

Having restricted $w$ and $v$ to be even/odd $2 \pi$-periodic functions of $t$, we thus consider the space $\Delta$, defined by

$$
\Delta=\left\{V(\cdot)=\left[\begin{array}{c}
w(\cdot) \\
v(\cdot)
\end{array}\right]: w, v \in L^{2}[0,2 \pi), w \text { even, } v \text { odd }\right\} .
$$

Then it follows that

$$
\mathcal{L}(y): \Delta \rightarrow \Delta \quad \text { and } \quad \mathcal{E}(y): \Delta \rightarrow \Delta
$$

and thus also

$$
\mathcal{M}: \Delta \rightarrow \Delta \text { and } \mathcal{N}: \Delta \rightarrow \Delta,
$$

and the eigenvalue problem should be posed in the space $\Delta$. Moreover, we have a natural orthogonal decomposition

$$
\Delta=\bigoplus_{n=0}^{+\infty} \Delta_{n}
$$

with respect to the $L^{2}$-inner product

$$
\left\langle V_{1}, V_{2}\right\rangle=\frac{1}{2 \pi} \int_{0}^{2 \pi} w_{1}(t) w_{2}(t)+v_{1}(t) v_{2}(t) d t,
$$

and where $\Delta_{n}$ is the $n$-th Fourier mode,

$$
\Delta_{n}=\left\{V_{n}(t)=\left[\begin{array}{c}
a_{n} \cos n t \\
b_{n} \sin n t
\end{array}\right]: a_{n}, b_{n} \in \mathbb{R}\right\} .
$$

This orthogonal decomposition is simply the Fourier cosine/sine series expansion of $V(\cdot)$. It is convenient to define the representation $\mathcal{T}_{n}^{*}: \mathbb{R}^{2} \rightarrow \Delta_{n}$ of the $n$-th mode by

$$
\mathcal{T}_{n}^{*}\left[\begin{array}{l}
a \\
b
\end{array}\right] \equiv\left[\begin{array}{c}
a \cos (n t) \\
b \sin (n t)
\end{array}\right]
$$


so a general $V \in \Delta$ has the Fourier decomposition

$$
V=\sum \mathcal{T}_{n}^{*}\left[\begin{array}{l}
a_{n} \\
b_{n}
\end{array}\right]
$$

corresponding to

$$
w(t)=\sum a_{n} \cos n t, \quad v(t)=\sum b_{n} \sin n t
$$

It is well known that the linear wave equation respects modes: in our notation, this is just the statement that the restriction of $\mathcal{L}(y)$ to $\Delta_{n}$ maps $\Delta_{n}$ back to itself. Indeed, the action of $\mathcal{L}(y)$ on $\Delta_{n}$ is rotation of the coefficients through angle $n y$. Also, it is clear that the operators $\mathcal{J}$ and $\mathcal{S}$ preserve the $n$-th mode, and we have the following theorem from [9]:

THEOREM 2. The linear operator $\mathcal{M}$ has the orthogonal representation

$$
\mathcal{M}=\oplus_{n=0}^{\infty} \mathcal{M}_{n}
$$

where $\mathcal{M}_{n}: \Delta_{n} \rightarrow \Delta_{n}$ is given by

$$
\mathcal{M}_{n}\left(\mathcal{T}_{n}^{*}\left[\begin{array}{l}
a_{n} \\
b_{n}
\end{array}\right]\right)=\mathcal{T}_{n}^{*}\left(M_{n}\left[\begin{array}{l}
a_{n} \\
b_{n}
\end{array}\right]\right)
$$

and where $M_{n}$ is the $2 \times 2$ matrix

$$
M_{n}=(-1)^{n} D^{-1} R(n \underline{\theta}) D R(n \bar{\theta}) \equiv(-1)^{n} H(n \bar{\theta}, n \underline{\theta}, J)
$$

here $R(\vartheta)$ is rotation by angle $\vartheta$,

$$
R(\vartheta)=\left(\begin{array}{cc}
\cos \vartheta & -\sin \vartheta \\
\sin \vartheta & \cos \vartheta
\end{array}\right)
$$

and $D$ is the diagonal matrix

$$
D=\left(\begin{array}{ll}
1 & 0 \\
0 & J
\end{array}\right)
$$

In (41), the rotations correspond to evolution, the diagonal matrix $D$ to the jump, and $(-1)^{n}$ to the shift of the $n$-th mode. We use the convention that script letters refer to operators, while capital letters refer to the matrices of their $2 \times 2$ components on $\Delta_{n}$. Because of the decomposition, the linearized eigenvalue problem (32) decouples into many $2 \times 2$ eigenvalue problems:

COROLlary 3. The function $V(t)$ given by (38) solves the linear eigenvalue problem (32) if and only if for all $n \in \mathbb{N}$,

$$
(-1)^{n} H(n \bar{\theta}, n \underline{\theta}, J)\left[\begin{array}{l}
a_{n} \\
b_{n}
\end{array}\right]=\left[\begin{array}{c}
a_{n} \\
b_{n}
\end{array}\right] .
$$


2.5. Periodic solutions to the linearized problem. Having reduced the problem of finding (linearized) periodic solutions to many $2 \times 2$ matrix eigenvalue problems (43), we now characterize these solutions. Because the matrix

$$
H(\bar{\vartheta}, \underline{\vartheta}, J) \equiv D^{-1}(J) R(\underline{\vartheta}) D(J) R(\bar{\vartheta})
$$

is explicitly given, we obtain exact formulae for its eigenvalues:

Theorem 4. For $J \neq 1$, the eigenvalues of $H(\bar{\vartheta}, \underline{\vartheta}, J)$ are

$$
\lambda=\beta \pm \sqrt{\beta^{2}-1}
$$

where

$$
\beta=\cos (\underline{\vartheta}) \cos (\bar{\vartheta})-\frac{J^{2}+1}{2 J} \sin (\underline{\vartheta}) \sin (\bar{\vartheta})
$$

It follows that in order for (43) to have a nontrivial solution, we must have $\lambda=\beta= \pm 1$, which represents a constraint on the parameters $(n \bar{\theta}, n \underline{\theta}, J)$. Our goal is to find a solution to the linearized problem which perturbs: we thus ask that (43) have a solution for $n=1$ only, the parameters $(\bar{\theta}, \underline{\theta}, J)$ remaining fixed: this is a nonresonance condition. First, based on the structure of solutions developed in [8], we restrict the range of parameters:

TheOrem 5. Assume that $J>1, \bar{\theta}>0, \underline{\theta}>0$ and

$$
\bar{\theta}+\underline{\theta}<\pi \text {. }
$$

Then $\mathbf{q}$ solves the eigenvalue problem

$$
H(\bar{\theta}, \underline{\theta}, J) \mathbf{q}=-\mathbf{q}
$$

if and only if

$$
J=\cot (\bar{\theta} / 2) \cot (\underline{\theta} / 2),
$$

and the solution is

$$
\mathbf{q} \in \operatorname{Span}\left\{\left[\begin{array}{c}
\cos (\bar{\theta} / 2) \\
-\sin (\bar{\theta} / 2)
\end{array}\right]\right\}
$$

In particular, if (49) holds, then

$$
V_{*}(t) \equiv \mathcal{T}_{1}^{*} \mathbf{q}=\left[\begin{array}{c}
\cos (\bar{\theta} / 2) \cos t \\
-\sin (\bar{\theta} / 2) \sin t
\end{array}\right]
$$

is a solution to the full eigenvalue problem (32).

2.6. Resonance and small divisors. We now consider the linear operator

$$
\mathcal{M}-\mathcal{I}: \Delta \rightarrow \Delta
$$

which also admits an orthogonal decomposition as in (39). A solution of (32) is an element of the kernel, and in order to perturb this, we'd like the kernel to be as small as 
possible. That is, we wish to minimize the number of solutions of (43). Since we have the constraint (49), we can accomplish this by choosing the parameters appropriately.

Here we calculate the full spectrum of $\mathcal{M}-\mathcal{I}$ and describe the nonresonance condition which will ensure that $\mathcal{M}-\mathcal{I}$ is invertible on higher modes. By Theorems 2 and 4 , the eigenvalues of $\mathcal{M}-\mathcal{I}$ are $\pm \lambda_{n}^{ \pm}-1$, where

$$
\lambda_{n}^{ \pm} \equiv \beta_{n} \pm \sqrt{\beta_{n}^{2}-1}
$$

with

$$
\begin{gathered}
\beta_{n} \equiv \beta_{n}(\underline{\theta}, \bar{\theta})=\beta(n \underline{\theta}, n \bar{\theta}), \quad \text { and } \\
\beta(\underline{\vartheta}, \bar{\vartheta}) \equiv \cos (\underline{\vartheta}) \cos (\bar{\vartheta})-\frac{J^{2}+1}{2 J} \sin (\underline{\vartheta}) \sin (\bar{\vartheta}) .
\end{gathered}
$$

The conditions $\lambda_{n} \neq \pm 1$ and $\beta_{n} \neq \pm 1$ are equivalent, and it is easier to work with $\beta_{n}$.

We obtain our nonresonance condition as follows. First, assuming $(\bar{\theta}, \underline{\theta})$ satisfies (47), we fix $J$ according to (49): this ensures that the kernel is nonempty. Now declare the parameters $(\bar{\theta}, \underline{\theta})$ to be resonant if

$$
\beta_{n}=(-1)^{n} \quad \text { for some } n>1 .
$$

It follows that at resonance, the kernel has a nontrivial component in the $n$-th mode, so we cannot invert the operator in that mode. On the other hand, if the parameters are nonresonant, then the kernel is invertible on all higher modes, and we would expect that our solution $V(t)$ may perturb.

Since there are countably many conditions $\beta_{n} \neq \pm 1$ but we have continuous parameters $\bar{\theta}$ and $\underline{\theta}$, we expect that most choices are nonresonant. In [9], we prove that the resonant set has measure zero:

THEOREM 6. Consider the parameter set

$$
E \equiv\{\Theta=(\bar{\theta}, \underline{\theta}): \bar{\theta}, \underline{\theta}>0,0<\bar{\theta}+\underline{\theta}<\pi\}
$$

Then there exists a set of full measure $E^{*} \subset E$ such that, if $\Theta \in E^{*}$, then $\Theta$ is nonresonant in the sense that when $J$ is given by (49), then the eigenvalues $\lambda_{n}^{ \pm}-(-1)^{n}$ of the linearized operator $\mathcal{M}-\mathcal{I}$ are nonzero for all $n \geq 2$.

Although $\mathcal{M}-\mathcal{I}$ is invertible on higher modes, it is not bounded: if it were bounded, we could apply the Implicit Function Theorem directly to obtain existence of periodic solutions. Instead, we have a problem of small divisors: the differences $\beta_{n}-(-1)^{n}$ accumulate at 0 as $n \rightarrow \infty$. Under the further symmetry assumption $\bar{\theta}=\underline{\theta}$, in [9] we were able to get lower bounds on the size of the small divisors:

THEOREM 7. Under the assumption $\bar{\theta}=\underline{\theta}=\theta$, there is a full measure set $E_{*} \subset(0,2 \pi)$, such that for every $\theta \in E_{*}$, there are positive constant $C$ and exponent $r \geq 1$ such that the eigenvalues satisfy the estimate

$$
\left|\lambda_{n}^{ \pm}-(-1)^{n}\right| \geq \frac{C}{n^{r}} \quad \text { for all } \quad n \geq 2 .
$$

In particular, if $\theta / \pi$ is the irrational root of a quadratic algebraic equation, we can take $r=1$. 
The assumption $\bar{\theta}=\underline{\theta}$ states that the different entropy levels have the same width in dimensionless variables ${ }^{3}$ : however, in standard variables the widths of the different entropy levels are given by (23), and so are different, although they do scale directly with the base sound speed.

3. Liapunov-Schmidt reduction. In this section we show that the nonlinear eigenvalue problem (1) can be recast as a perturbation from the linear eigenvalue problem (2). We then show that the resulting bifurcation problem is amenable to a Liapunov-Schmidt reduction [4]. We perform the reduction in the case of arbitrary non-resonant, non-symmetric periods $\Theta=(\bar{\theta}, \underline{\theta})$, as in Theorem 6 . This reduces the problem of the existence of solutions of (1) to the problem of proving an implicit function theorem for the so called auxiliary equation, an equation that asks for the existence of nearby zeros of a nonlinear operator in a neighborhood of the zero of an invertible linearized operator. The invertible linearized operator is the restriction of (2) to the complement of the solution kernel, and has small divisors. The invertibility in the case of general non-resonant, non-symmetric $\Theta$ is a direct consequence of Theorem 6 , and for symmetric $\Theta=(\theta, \theta)$, algebraic estimates for the small divisors for almost every $\theta$ follow directly from Theorem 7 . The implicit function theorem posed by the auxiliary equation for (1) is a KAM type small divisor problem in an infinite dimensional space based on a nonlinear differential operator of quasilinear type. As far as we know, a complete mathematical proof of this implicit function theorem is beyond the direct application of known results, although analogous results have been obtained for similar problems of semi-linear type, c.f. [2]. The purpose of this analysis is to prove that the (finite dimensional) implicit function theorem posed by the bifurcation equation is valid assuming existence of solutions for the (infinite dimensional) implicit function theorem associated with the auxiliary equation.

In Section 5 we show that the Liapunov-Schmidt reduction that applies in the infinite dimensional case also applies when (1) is replaced by a Fourier cutoff of the nonlinear problem up to arbitrarily high Fourier modes. In the case of an $N$-Fourier mode cutoff, $N$ arbitrarly large, we prove that the resulting auxiliary equation has a unique solution, confirming that the two applications of the implicit function theorem inherent in the Liapunov-Schmidt method are both valid for every $N \geq 2$. This provides strong evidence that periodic solutions of the compressible Euler equations exhibiting the wave structure that balances compression and rarefaction described at the linearized level in [9] should exist.

3.1. Coordinates and notation. We now develop the pertubation problem and establish notation and preliminary lemmas required to obtain the auxiliary and bifurcation equations associated with the Liapunov-Schmidt reduction of the infinite dimensional problems (1), (2).

Recall that the operators $\mathcal{M}$ and $\mathcal{N}$ map $2 \pi$-periodic functions of $t$ (at $y=0$ ) to $2 \pi$-periodic functions of $t$ (at $y=\bar{\theta}+\underline{\theta}$ ),

$$
\mathcal{M}: \Delta \rightarrow \Delta \text { and } \mathcal{N}: \Delta \rightarrow \Delta
$$

where $\Delta$ is the function space of real valued $2 \pi$-periodic functions that are even in $w$ and odd in $v$. By the $H^{s}$ estimates for smooth solutions of conservation laws [5], it follows that both operators $\mathcal{M}$ and $\mathcal{N}$ take sufficiently smooth and sufficiently small

\footnotetext{
${ }^{3}$ In [9] the use of one variable yields explicit formulas for $\beta_{n}-1$ which can be directly estimated; we expect a similar result holds for two variables, but this may require a probabilistic proof. This is an ongoing topic of research by the authors and Roman Vershynin.
} 
functions in $H^{s} \cap \Delta$ to $H^{s} \cap \Delta$, for any $s>3 / 2$, so we will not need to be concerned about shock wave formation for sufficiently small data.

In the Liapunov-Schmidt method, bifurcation to nonlinear solutions is analyzed in coordinates induced on the solution space by the structure of the linearized operator [4, 3]. For the full infinite dimensional problem (1) the solution space is $\Delta$ and the linearized operator is $\mathcal{M}-\mathcal{I}$. Also, according to (34),

$$
\Delta=\oplus_{n \geq 0} \Delta_{n}
$$

where $\Delta_{n}$ is given by (36), so that $\Delta_{n}$ is two dimensional for $n \geq 1$, and one dimensional in the special case $n=0$. That is, for $V \in \Delta$, we write

$$
V(t)=\sum_{n=0}^{\infty} V_{n}(t)=\sum_{n=0}^{\infty} \mathcal{T}_{n}^{*} \mathbf{p}_{n}
$$

where $\mathbf{p}_{n}=\left(a_{n}, b_{n}\right) \in \mathbb{R}^{2}$, so $V_{n} \in \Delta_{n}$ is given by

$$
V_{n}(t)=\mathcal{T}_{n}^{*} \mathbf{p}_{n}=\left[\begin{array}{c}
a_{n} \cos n t \\
b_{n} \sin n t
\end{array}\right]
$$

and $\mathcal{T}_{n}^{*}: \mathbb{R}^{2} \rightarrow \Delta_{n}$ is defined in (37). Referring to (35), we can take the $L^{2}$-inner product on $\Delta$ to be given by

$$
\left\langle V, V^{\prime}\right\rangle=\sum_{n=0}^{\infty} \mathbf{p}_{n} \cdot \mathbf{p}_{n}^{\prime} \equiv \sum_{n=0}^{\infty} a_{n} a_{n}^{\prime}+b_{n} b_{n}^{\prime},
$$

for

$$
V=\sum_{n=0}^{\infty} \mathcal{T}_{n}^{*} \mathbf{p}_{n} \in \Delta, \quad V^{\prime}=\sum_{n=0}^{\infty} \mathcal{T}_{n}^{*} \mathbf{p}_{n}^{\prime} \in \Delta
$$

We now characterize the kernel and range of $\mathcal{M}_{1}-\mathcal{I}$ and $\mathcal{M}-\mathcal{I}$ in $\Delta$, respectively. Referring to Thm. 5, we set

$$
\mathbf{q}=\left[\begin{array}{c}
\cos (\bar{\theta} / 2) \\
-\sin (\bar{\theta} / 2)
\end{array}\right], \quad \text { and } \quad \mathbf{q}^{\perp}=\left[\begin{array}{c}
\sin (\bar{\theta} / 2) \\
\cos (\bar{\theta} / 2)
\end{array}\right],
$$

so that

$$
V_{*}(t)=\mathcal{T}_{n}^{*} \mathbf{q}
$$

The first lemma gives a precise characterization of the map $M_{1}-I$.

Lemma 8. The $2 \times 2$ matrix $M_{1}-I$ can be expressed as

$$
M_{1}-I=\tau_{0} \mathbf{q} \otimes \mathbf{q}^{\perp} \equiv \tau_{0} \mathbf{q}\left(\mathbf{q}^{\perp}\right)^{t},
$$

where the constant $\tau_{0}$ is given by

$$
\left.\tau_{0} \equiv \tau_{0}(\bar{\theta}), \underline{\theta}\right)=\sin \bar{\theta} \cos \underline{\theta}+\left(J \cos ^{2} \bar{\theta} / 2-\frac{1}{J} \sin ^{2} \bar{\theta} / 2\right) \sin \underline{\theta},
$$


with $J=\cot \bar{\theta} / 2 \cot \underline{\theta} / 2$ given by (49). Moreover, in the non-symmetric case, there exists a set of full measure $E_{0} \subset E$ such that

$$
\tau_{0}(\bar{\theta}, \underline{\theta}) \neq 0 \quad \text { for all } \quad(\bar{\theta}, \underline{\theta}) \in E_{0} ;
$$

and for the symmetric case we have

$$
\tau_{0}(\theta, \theta) \neq 0 \text { for all } \theta \in(0, \pi / 2) .
$$

Proof. Since $M_{1}-I$ has rank one, it is of the form $\mathbf{p}_{1} \otimes \mathbf{p}_{2}$ for some vectors $\mathbf{p}_{i} \in \mathbb{R}^{2}$. Since $\mathbf{q}$ is in the kernel, $\mathbf{p}_{2}=k_{2} \mathbf{q}^{\perp}$, and since the range is $\operatorname{Span}\{\mathbf{q}\}$, we have $\mathbf{p}_{2}=k_{1} \mathbf{q}$. Thus (59) holds, and since $\mathbf{q}$ and $\mathbf{q}^{\perp}$ are unit vectors, we have

$$
\tau_{0}=\mathbf{q} \cdot\left(M_{1}-I\right) \mathbf{q}^{\perp}=\mathbf{q}^{t} M_{1} \mathbf{q}^{\perp} .
$$

Now by (40), we have

$$
M_{1}=-D^{-1} R(\underline{\theta}) D R(\bar{\theta})
$$

and using (58), we calculate

$$
R(\bar{\theta}) \mathbf{q}^{\perp}=R(\bar{\theta}+\pi / 2) \mathbf{q}=\left[\begin{array}{c}
-\sin \bar{\theta} / 2 \\
\cos \bar{\theta} / 2
\end{array}\right] .
$$

It follows that

$$
\begin{aligned}
\tau_{0} & =\left[\begin{array}{c}
-\cos \bar{\theta} / 2 \\
\sin \bar{\theta} / 2
\end{array}\right]^{t}\left(\begin{array}{cc}
\cos \underline{\theta} & -J \sin \underline{\theta} \\
\sin \underline{\theta} / J & \cos \underline{\theta}
\end{array}\right)\left[\begin{array}{c}
-\sin \bar{\theta} / 2 \\
\cos \bar{\theta} / 2
\end{array}\right] \\
& =\cos \underline{\theta}(2 \cos \bar{\theta} / 2 \sin \bar{\theta} / 2)+\sin \underline{\theta}\left(J \cos ^{2} \bar{\theta} / 2-\frac{1}{J} \sin ^{2} \bar{\theta} / 2\right),
\end{aligned}
$$

which is $(60)$.

To verify (61), substitute (49) into (60) to write $\tau_{0}$ as a trigonometric polynomial in $(\bar{\theta}, \underline{\theta})$, which is zero on a set of measure zero, whose complement in $E^{*}$ defines $E_{0}$. For (62), set $\bar{\theta}=\theta=\theta$, and use $J=\cot ^{2} \theta / 2$ together with the identities $\cos ^{2} \theta / 2, \sin ^{2} \theta / 2=\frac{1}{2}(1 \pm \cos \theta)$, respectively, to obtain

$$
\tau_{0}(\theta, \theta)=\sin \theta \cos \theta+\frac{(1+\cos \theta)^{3}-(1-\cos \theta)^{3}}{2 \sin \theta},
$$

which is non-zero when $\cos \theta$ and $\sin \theta$ are both positive. $\square$

The next lemma characterizes the kernel and range of $\mathcal{M}-\mathcal{I}$ in $\Delta$.

Lemma 9. Assume that $\Theta=(\bar{\theta}, \underline{\theta}) \in E_{0}$, so $\Theta$ is non-resonant and $\tau_{0} \neq 0$. Then the kernel $\mathcal{K}$ of $\mathcal{M}-\mathcal{I}$ in $\Delta$ is the 2-dimensional subspace

$$
\mathcal{K}=\Delta_{0} \oplus \operatorname{Span}\left\{\mathcal{T}_{1}^{*} \mathbf{q}\right\},
$$

and the range $\mathcal{R}$ of $\mathcal{M}-\mathcal{I}$ is the subspace

$$
\mathcal{R}=\operatorname{Span}\left\{\mathcal{T}_{1}^{*} \mathbf{q}\right\} \oplus\left\{\oplus_{n=2}^{\infty} \Delta_{n}\right\} .
$$

In the symmetric case $\bar{\theta}=\underline{\theta}=\theta$, both (64) and (65) hold for all $\theta \in E_{*}$, where $E_{*} \subset(0,2 \pi)$ is the set on which (56) holds. 
Proof. By (40), it follows immediately that $M_{0}=I$, so that $\Delta_{0} \subset \mathcal{K}$, and by Theorem 5 ,

$$
V_{*}(t)=\mathcal{T}_{1}^{*} \mathbf{q} \in \mathcal{K} .
$$

By Theorem $6, M_{n}-I$ is invertible for each $n>1$, so that $\oplus_{n=2}^{\infty} \Delta_{n} \subset \mathcal{R}$ and these contribute nothing to the kernel. To complete the proof, it thus suffices to show that the range of the matrix $M_{1}-I$ is $\operatorname{Span}\{\mathbf{q}\}$. But by Lemma 8 , this is exactly the condition that $\tau_{0} \neq 0$, which holds because we have assumed $\Theta \in E_{0}$ or $\theta \in E_{*}$.

It is convenient to introduce refined notation for the kernel as a 1-mode subspace. Thus let $\mathcal{K}^{\perp}$ denote the orthogonal complement of $\mathcal{K}$ in $\Delta$,

$$
\mathcal{K}^{\perp}=\mathcal{K}_{1}^{\perp} \oplus\left\{\oplus_{n=2}^{\infty} \Delta_{n}\right\},
$$

where the 1-mode part of the kernel is denoted by

$$
\mathcal{K}_{1} \equiv \mathcal{K} \cap \Delta_{1}=\operatorname{Span}\left\{\mathcal{T}_{1}^{*} \mathbf{q}\right\}
$$

and its orthogonal complement in $\Delta_{1}$ is

$$
\mathcal{K}_{1}^{\perp} \equiv \mathcal{K}^{\perp} \cap \Delta_{1}=\operatorname{Span}\left\{\mathcal{T}_{1}^{*} \mathbf{q}^{\perp}\right\}
$$

Similarly, let $\mathcal{R}^{\perp}$ be the orthogonal complement of $\mathcal{R}$,

$$
\mathcal{R}^{\perp}=\Delta_{0} \oplus \mathcal{K}_{1}^{\perp}
$$

and also define

$$
\mathcal{P}: \Delta \rightarrow \Delta
$$

to be orthogonal projection onto the range $\mathcal{R}$. The next lemma implies that the infinite dimensional operator $\mathcal{P}(\mathcal{M}-\mathcal{I})$, restricted to $\mathcal{K}^{\perp}$, is an invertible operator, provided $\Theta$ is nonresonant:

LEMMA 10. If $\Theta \in E_{0}$ is nonresonant then the operator

$$
\mathcal{P}(\mathcal{M}-\mathcal{I}): \mathcal{K}^{\perp} \rightarrow \mathcal{R}
$$

admits the decomposition

$$
\mathcal{P}(\mathcal{M}-\mathcal{I})=\mathcal{P}_{1}\left(\mathcal{M}_{1}-\mathcal{I}\right) \oplus\left\{\oplus_{n=2}^{\infty}\left(\mathcal{M}_{n}-\mathcal{I}\right)\right\},
$$

and $\mathcal{P}(\mathcal{M}-\mathcal{I}): \mathcal{K}^{\perp} \rightarrow \mathcal{R}$ is invertible. In this case the 1 -mode projection

$$
\mathcal{P}_{1}\left(\mathcal{M}_{1}-\mathcal{I}\right): \mathcal{K}_{1}^{\perp} \rightarrow \mathcal{K}_{1}
$$

is multiplication by $\tau_{0} \neq 0$, in the sense that for any $X_{1} \in \mathbb{R}$,

$$
\left(\mathcal{M}_{1}-\mathcal{I}\right)\left(X_{1} \mathcal{T}_{1}^{*} \mathbf{q}^{\perp}\right)=\tau_{0} X_{1} \mathcal{T}_{1}^{*} \mathbf{q}
$$

Moreover, for $n \geq 2$, the eigenvalues of the $n$-th mode decomposition are $(-1)^{n} \lambda_{n}^{ \pm}-1$, where $\lambda_{n}^{ \pm}$are given by (52).

In the symmetric case $\bar{\theta}=\underline{\theta}=\theta \in E_{*} \subset(0, \pi / 2)$, we have $\tau_{0} \neq 0$, and there exist constants $C=C(\theta)>0$ and exponent $r$ such that for $n \geq 2$, these eigenvalues satisfy the lower bound

$$
\left|(-1)^{n} \lambda_{n}^{ \pm}-1\right| \geq \frac{C(\theta)}{n^{r}}
$$


Proof. The decomposition follows directly from Lemmas 9 and 8 above. The 1-mode projection $\mathcal{P}_{1}\left(\mathcal{M}_{1}-\mathcal{I}\right)$ is invertible for $\tau_{0} \neq 0$, and (71) holds for $n=1$ provided $C(\theta)<\left|\tau_{0}\right|$. The eigenvalues follow directly from (40) and (52), and the estimate (71) follows from (56) of Theorem 7 . Finally, by $(71)$, each $\mathcal{P}(\mathcal{M}-\mathcal{I})$ is invertible (i.e. one-to-one and onto) on each of the spaces in the decomposition, so that the full operator $\mathcal{P}(\mathcal{M}-\mathcal{I})$ is invertible as well.

Our ultimate goal is to prove the existence of solutions of the nonlinear problem $(\mathcal{N}-\mathcal{I})[V]=0$ that bifurcate from solutions of the associated linearized problem $(\mathcal{M}-\mathcal{I})[V]=0$, where the initial data $V \in \Delta$ is a $2 \pi$-periodic function of $t$, with values $V(t)$. To set this up as a perturbation problem, we will introduce a small parameter $-1<<\epsilon<<1$ that measures the strength of nonlinear perturbation from the linearized problem, and which will act as the nonlinear bifurcation parameter. To provide more flexibility in the bifurcation argument, we now also introduce additional small parameters

$$
\alpha=\left(\bar{\alpha}, \underline{\alpha}, \alpha_{J}\right), \quad \text { with } \quad-1 \ll \bar{\alpha}, \underline{\alpha}, \alpha_{J} \ll 1,
$$

that represent allowable changes in the entropy widths $\bar{\theta}$ and $\underline{\theta}$ and jump $J$ for the bifurcating solutions. This is analogous to the variation of the period when treat Hopf bifurcations [4].

We thus define the linear and nonlinear operators

$$
\begin{aligned}
\mathcal{M}_{\alpha} & \equiv \mathcal{S} \cdot \mathcal{J}_{\alpha}^{-1} \cdot \mathcal{L}(\underline{\theta}+\underline{\alpha}) \cdot \mathcal{J}_{\alpha} \cdot \mathcal{L}(\bar{\theta}+\bar{\alpha}), \\
\mathcal{N}_{\alpha} & \equiv \mathcal{S} \cdot \mathcal{J}_{\alpha}^{-1} \cdot \mathcal{E}(\underline{\theta}+\underline{\alpha}) \cdot \mathcal{J}_{\alpha} \cdot \mathcal{E}(\bar{\theta}+\bar{\alpha})
\end{aligned}
$$

as well as the $2 \times 2$ real matrix

$$
M_{\alpha}=-D_{\alpha}^{-1} R(\underline{\theta}+\underline{\alpha}) D_{\alpha} R(\bar{\theta}+\bar{\alpha}),
$$

used to represent $\mathcal{M}_{\alpha}$, in analogy with (31). Here $\mathcal{J}_{\alpha}$ is the perturbation of the jump operator defined in (25) with matrix $D_{\alpha}$ obtained by replacing $J$ with $J+\alpha_{J}$,

$$
D_{\alpha}=\left(\begin{array}{cc}
1 & 0 \\
0 & J+\alpha_{J}
\end{array}\right), \quad D_{\alpha}^{-1}=\left(\begin{array}{cc}
1 & 0 \\
0 & \frac{1}{J+\alpha_{J}}
\end{array}\right),
$$

so that $D_{0} \equiv D$ when $\alpha_{J}=0$, see (42).

Now the operators $\mathcal{M}_{\alpha}, \mathcal{N}_{\alpha}$, as well as the operators $\mathcal{L}(\cdot), \mathcal{E}(\cdot)$ used to define them, all operate on initial data $V \in \Delta$ in the domain of the given operator at $y=0$, and output the result $V(y)$ of an evolution in $\Delta$ of functions indexed by $y$. For such an operator $\mathcal{O}$ let its domain be denoted $\Delta_{\mathcal{O}}$,

$$
\mathcal{O}: \Delta_{\mathcal{O}} \rightarrow \Delta
$$

and use the following notation. If the input $V \in \Delta$ is given by

$$
V:[0,2 \pi] \rightarrow \mathbb{R}^{2}, \quad V(t)=\left[\begin{array}{c}
w(t) \\
v(t)
\end{array}\right] \in \mathbb{R}^{2},
$$

we denote the output as $\mathcal{O}[V] \in \Delta$,

$$
\mathcal{O}[V]:[0,2 \pi] \rightarrow \mathbb{R}^{2},
$$


the function of $t$ with values $\mathcal{O}[V](t)$. Further, if the function $\mathcal{O}[V]$ is the result of evolution from $y=y_{0}$ to $y=y_{1}$, then we define

$$
\mathcal{O}(y)[V] \equiv \mathcal{O}[V](y) \in \Delta, \quad \text { for } \quad y_{0} \leq y \leq y_{1},
$$

as that function in $\Delta$ determined at the value $y$ of the evolution, with values $V(y, t)$. Here $V(y, t)$ is the solution of the PDE with data $V\left(y_{0}, t\right)$, and $\mathcal{O}[V]$ has values $V\left(y_{1}, t\right)$.

Thus, in particular, the full solution of the (perturbed) linear and nonlinear partial differential equations are described by

$$
\mathcal{M}_{\alpha}(y)[V] \equiv \begin{cases}\mathcal{L}(y)[V], & 0 \leq y<\bar{\theta}+\bar{\alpha}, \\ \mathcal{L}(y-\bar{\theta}-\bar{\alpha}) \mathcal{J}_{\alpha} \mathcal{L}(\bar{\theta}+\bar{\alpha})[V], & 0<y-\bar{\theta}-\bar{\alpha}<\underline{\theta}+\underline{\alpha}, \\ \mathcal{S} \mathcal{J}_{\alpha}^{-1} \mathcal{L}(\underline{\theta}+\underline{\alpha}) \mathcal{J}_{\alpha} \mathcal{L}(\bar{\theta}+\bar{\alpha})[V], & y=\bar{\theta}+\bar{\alpha}+\underline{\theta}+\underline{\alpha},\end{cases}
$$

and

$$
\mathcal{N}_{\alpha}(y)[V] \equiv \begin{cases}\mathcal{E}(y)[V], & 0 \leq y<\bar{\theta}+\bar{\alpha}, \\ \mathcal{E}(y-\bar{\theta}-\bar{\alpha}) \mathcal{J}_{\alpha} \mathcal{E}(\bar{\theta}+\bar{\alpha})[V], & 0<y-\bar{\theta}-\bar{\alpha}<\underline{\theta}+\underline{\alpha}, \\ \mathcal{S} \mathcal{J}_{\alpha}^{-1} \mathcal{E}(\underline{\theta}+\underline{\alpha}) \mathcal{J}_{\alpha} \mathcal{E}(\bar{\theta}+\bar{\alpha})[V], & y=\bar{\theta}+\bar{\alpha}+\underline{\theta}+\underline{\alpha},\end{cases}
$$

respectively, each $\mathcal{M}_{\alpha}(y)[V]$ and $\mathcal{N}_{\alpha}(y)[V] \in \Delta$ being a function of $t$. Note that the functions have well-defined left and right limits at the discontinuity $y=\bar{\theta}+\bar{\alpha}$.

Finally, define the matrix representation $M_{\alpha}(y)$ of the operator $\mathcal{M}_{\alpha}(y)$ restricted to the 1-mode by using the same identity as before, namely

$$
\mathcal{M}_{\alpha}(y)\left[\mathcal{T}_{1}^{*} \mathbf{p}\right] \equiv \mathcal{T}_{1}^{*}\left[M_{\alpha}(y) \mathbf{p}\right],
$$

which leads to the explicit formula

$$
M_{\alpha}(y)= \begin{cases}R(y), & 0 \leq y<\bar{\theta}+\bar{\alpha}, \\ R(y-\bar{\theta}-\bar{\alpha}) D_{\alpha} R(\bar{\theta}+\bar{\alpha}), & 0<y-\bar{\theta}-\bar{\alpha}<\underline{\theta}+\underline{\alpha}, \\ -D_{\alpha}^{-1} R(\underline{\theta}+\underline{\alpha}) D_{\alpha} R(\bar{\theta}+\bar{\alpha}), & y=\bar{\theta}+\bar{\alpha}+\underline{\theta}+\underline{\alpha},\end{cases}
$$

and in particular, by (73), $M_{\alpha}(\bar{\theta}+\bar{\alpha}+\underline{\theta}+\underline{\alpha}) \equiv M_{\alpha}$.

3.2. Rescaling and reduction. We now introduce the scaling parameter $\epsilon$ into the nonlinear operator $\mathcal{N}$ so that the nonlinear problem (1) reduces to the linear eigenvalue problem (2) at $\epsilon=0$. Without loss of generality, we linearize the system (20) around the trivial solution consisting of the base state

$$
w(y, t)=1, \quad v(y, t)=0,
$$

and we denote this base state by

$$
\mathbf{1} \equiv\left(\begin{array}{l}
1 \\
0
\end{array}\right) \in \mathbb{R}^{2} \quad \text { so that } \quad\left[\begin{array}{c}
w \\
v
\end{array}\right]=\mathcal{T}_{0}^{*} \mathbf{1} .
$$

For $\epsilon \neq 0$, define the rescaled nonlinear operator $\mathcal{G}_{\epsilon, \alpha}: \Delta \rightarrow \Delta$ by

$$
\mathcal{G}_{\epsilon, \alpha}[V]=\frac{1}{\epsilon}\left\{\mathcal{N}_{\alpha}[\mathbf{1}+\epsilon V]-\mathbf{1}\right\},
$$

and the corresponding operator $\mathcal{F}_{\epsilon, \alpha}: \Delta \rightarrow \Delta$ by

$$
\mathcal{F}_{\epsilon, \alpha}=\mathcal{G}_{\epsilon, \alpha}-\mathcal{I},
$$


so that

$$
\mathcal{F}_{\epsilon, \alpha}[V]=\mathcal{G}_{\epsilon, \alpha}[V]-V
$$

By Theorem 1, we need only construct solutions of $(\mathcal{N}-\mathcal{I})[V]=0$ for $V(t)=$ $(w(t), v(t))$ satisfying the average one in $w$ and zero in $v$ conditions (28), respectively, in order to obtain solutions with arbitrary averages (29) in the physical variables.

LEMma 11. The nonlinear operator $\mathcal{G}_{\epsilon, \alpha}$ has the limits

$$
\lim _{\epsilon \rightarrow 0} \mathcal{G}_{\epsilon, \alpha} \equiv \mathcal{G}_{\alpha}=\mathcal{M}_{\alpha}
$$

and

$$
\lim _{\epsilon, \alpha \rightarrow 0} \mathcal{G}_{\epsilon, \alpha} \equiv \mathcal{G}_{0}=\mathcal{M}
$$

in the sense that

$$
\begin{aligned}
& \mathcal{G}_{\epsilon, \alpha}(y)[V]=\mathcal{M}_{\alpha}(y)[V]+O(|\epsilon|), \\
& \mathcal{G}_{\epsilon, \alpha}(y)[V]=\mathcal{M}(y)[V]+O(|\epsilon|+|\alpha|),
\end{aligned}
$$

for all smooth, $2 \pi$-periodic initial data $V \in \Delta$, which take on values $V(t)$, and where convergence is uniform for

$$
(y, t) \in[0, \bar{\theta}+\bar{\alpha}+\underline{\theta}+\underline{\alpha}] \times[0,2 \pi] .
$$

We thus also have, in the same sense,

$$
\lim _{\epsilon \rightarrow 0} \mathcal{F}_{\epsilon, \alpha} \equiv \mathcal{F}_{\alpha}=\mathcal{M}_{\alpha}-\mathcal{I}
$$

and

$$
\lim _{\epsilon, \alpha \rightarrow 0} \mathcal{F}_{\epsilon, \alpha} \equiv \mathcal{F}_{0}=\mathcal{M}-\mathcal{I}
$$

Proof. Since for fixed $\Theta$ and $\alpha$, the operators $\mathcal{J}_{\alpha}, \mathcal{J}_{\alpha}^{-1}$ and $\mathcal{S}$ are bounded and linear, the Lemma follows if we show that

$$
\lim _{\epsilon \rightarrow 0} \frac{1}{\epsilon}\{\mathcal{E}(y)[\mathbf{1}+\epsilon V]-\mathbf{1}\}=\mathcal{L}(y)[V],
$$

uniformly in $(y, t)$ for $y$ in a compact interval. As $\epsilon \rightarrow 0$, the values of the initial data

$$
U_{\epsilon} \equiv \mathbf{1}+\epsilon V
$$

tend to the constant state $(w, v)=(1,0)$. Thus the local existence theorem for smooth solutions of the nonlinear problem (20) implies that for $\epsilon$ sufficiently small, the output $\mathcal{E}(y)\left[U_{\epsilon}\right]$ is a well defined function, $2 \pi$-periodic in $t$, whose values tend to the constant state $(w, v)=(1,0)$ as $\epsilon \rightarrow 0$, uniformly for $(y, t) \in[0, \bar{\theta}+\bar{\alpha}+\underline{\theta}+\underline{\alpha}] \times[0,2 \pi]$, see [5]. This follows directly from the $H^{s}$ estimates for smooth solutions of the nonlinear problem (20), provided $\Delta \subset H^{s}$, [5]. Here, by (25), the jump operators $\mathcal{J}_{\alpha}$ and $\mathcal{J}_{\alpha}^{-1}$ fix $w$ and scale $v$ by a constant amount, and thus only change the constants in the $H^{s}$ estimates. 
More precisely, since $\mathcal{N}_{\alpha}(y)\left[U_{\epsilon}\right]$ is smooth and tends uniformly to $(1,0)$, it follows that the wavespeed $\sigma\left(w_{\epsilon, \alpha}(y, t)\right) \rightarrow 1$ uniformly as $\epsilon \rightarrow 0$, and hence that the characteristics of the nonlinear evolution (20) differ by at most order $\epsilon$ from the characteristics of the linear evolution (30) as $\epsilon \rightarrow 0$. From this it is clear that nonlinear evolution by $\mathcal{N}_{\alpha}$ is linear evolution by $\mathcal{M}_{\alpha}$ to leading order in $\epsilon$. Therefore,

$$
\begin{aligned}
\mathcal{N}_{\alpha}(y)\left[U_{\epsilon}\right] & =\mathcal{M}_{\alpha}(y)\left[U_{\epsilon}\right]+O\left(\epsilon^{2}\right) \\
& =\mathbf{1}+\epsilon \mathcal{M}_{\alpha}(y)[V]+O\left(\epsilon^{2}\right) .
\end{aligned}
$$

The Lemma follows because everything is smooth and convergence is uniform on compact sets.

Our goal now is to prove the existence of solutions $V_{\epsilon, \alpha} \in \Delta$ of the equation

$$
\mathcal{F}_{\epsilon, \alpha}\left[V_{\epsilon, \alpha}\right]=0,
$$

with $\epsilon \neq 0$, and $V_{\epsilon, \alpha}$ nontrivial, that is, $V_{\epsilon, \alpha} \notin \Delta_{0}$. Such a solution would clearly satisfy

$$
\mathcal{N}_{\alpha}\left[1+\epsilon V_{\epsilon, \alpha}\right]=1+\epsilon V_{\epsilon, \alpha},
$$

which by Theorem 1 (with perturbed $\Theta$ and $J$ ) yields a nontrivial periodic solution to the Euler equations.

To obtain solutions of (79) by the Liapunov Schmidt method, we need to apply the implicit function theorem twice to prove that solutions $V_{\epsilon, \alpha}$ of (79) bifurcate from known solutions $V \in \mathcal{K}$ of

$$
\mathcal{F}_{0,0} V=(\mathcal{M}-\mathcal{I}) V=0 .
$$

Before establishing the requisite auxiliary and bifurcation equations carefully, we first outline again the overall strategy in terms of our present notation. By Lemma 11, for small $\epsilon($ and $\alpha)$ we have

$$
\mathcal{F}_{\epsilon, \alpha} \approx \mathcal{M}-\mathcal{I}
$$

which is invertible on $\mathcal{K}^{\perp}$. Assuming we can invert $\mathcal{M}-\mathcal{I}$ on $\mathcal{K}^{\perp}$, it follows that because $\Delta=\mathcal{K} \oplus \mathcal{K}^{\perp}$, we need to solve $\operatorname{dim}(\mathcal{K})=2$ more equations to get a periodic solution.

Our procedure, then, for analyzing the 0 - and 1-modes in the kernel $\mathcal{K}$ is as follows: Using the invertibility of $\mathcal{M}-\mathcal{I}$ on $\mathcal{K}^{\perp}$, a first application of the implicit function theorem (in infinite dimensions) means that there is some $V=V_{\epsilon, \alpha}$ such that $\mathcal{F}_{\epsilon, \alpha}\left[V_{\epsilon, \alpha}\right]$ vanishes on $\mathcal{K}^{\perp}$. That is, we can take the $n$-mode of $\mathcal{F}_{\epsilon, \alpha}\left[V_{\epsilon, \alpha}\right]$ to be zero for all $n \geq 2$. This is equivalent to assuming the existence of a solution of the auxiliary equation, as expressed in Definition 12 below. Assuming a solution of the auxiliary equation, a second application of the implicit function theorem (in finite dimensions) shows that among such $V_{\epsilon, \alpha}$, the 1-mode of $\mathcal{F}_{\epsilon, \alpha}\left[V_{\epsilon, \alpha}\right]$ vanishes near $(\epsilon, \alpha)=0 \in \mathbb{R}^{4}$. This occurs along a (3-d) surface $\psi\left(\epsilon, \bar{\alpha}, \underline{\alpha}, \alpha_{J}\right)=0$, for some smooth function $\psi: \mathbb{R}^{4} \rightarrow \mathbb{R}$ with $\psi(0)=0$. This is accomplished in Theorem 13 by solving the bifurcation equation, and we prove this for almost every $\Theta$, assuming the existence of solutions of the auxiliary equation.

It then remains to prove that the zero mode of $\mathcal{F}_{\epsilon, \alpha}\left[V_{\epsilon, \alpha}\right]$ is zero. Since we are not using a conservative form of the nonlinear equations, the zero mode is not in general 
constant for the nonlinear problem. However, we can use the conservation form of the nonlinear equations to derive an integral that is conserved under nonlinear evolution. We then show that if all of the modes $n \geq 1$ of $\mathcal{F}_{\epsilon, \alpha}\left[V_{\epsilon, \alpha}\right]$ are zero and the integral is conserved, then the zero mode is also necessarily zero ${ }^{4}$.

Putting these together we obtain a proof of Theorem 14, which states that if $\Theta=(\bar{\theta}, \underline{\theta})$ is nonresonant and an appropriate solution of the auxiliary equation exists, then a three parameter family $V_{\epsilon, \alpha}$ of solutions bifurcates from the solution $\mathcal{T}_{1}^{*} \mathbf{q}$ of the linearized problem. In the final section we show that the assumed existence of a solution of the auxiliary equation is valid under the $N$-Fourier cutoff assumption, and the rest of the argument goes through essentially unchanged.

To make this more precise, fix a solution to the linearized problem $Z \in \mathcal{K}_{1}$, from which our solution bifurcates. For convenience, we take $Z \in \mathcal{K}$ to be given by (51),

$$
Z \equiv \mathcal{T}_{1}^{*} \mathbf{q}=V_{*},
$$

and which has values

$$
Z(t)=V_{*}(t)=\left[\begin{array}{c}
\cos (\bar{\theta} / 2) \cos t \\
-\sin (\bar{\theta} / 2) \sin t
\end{array}\right] .
$$

There is no loss of generality here as any element $Z_{1}=X_{1} V_{*}$ can be scaled back to $Z$ by the substitution $\epsilon \rightarrow X_{1} \epsilon$. Also note that we have set the component of $Z(t)$ in the zero mode kernel $\Delta_{0}$ of $\mathcal{M}-\mathcal{I}$ equal to zero, because the inclusion of $(w, v)=(1,0)$ in the definition of $\mathcal{F}$ already fixes the zero mode. However, this constant 0 -mode will not be preserved under nonlinear evolution, since we are not working with a conservative form of the nonlinear equations.

Now let $B_{\delta} \subset \mathbb{R}^{3}$ denote the ball of radius $\delta$, and $I_{\delta}$ the interval $(-\delta, \delta)$. We can then restate our problem as follows: Find $\delta_{1}, \delta_{2}>0$ and smooth functions

$$
W=W_{\epsilon, \alpha}: I_{\delta_{1}} \times B_{\delta_{1}} \rightarrow \mathcal{K}^{\perp} \quad \text { and } \quad \alpha=\alpha(\epsilon): I_{\delta_{2}} \rightarrow B_{\delta_{1}} \subset \mathbb{R}^{3},
$$

with $\alpha(0)=0, W_{0,0}=0$, such that for $\epsilon<\delta_{2}$, we have

$$
\mathcal{F}_{\epsilon, \alpha(\epsilon)}\left[Z+W_{\epsilon, \alpha(\epsilon)}\right]=0 \text {. }
$$

The Liapunov-Schmidt method reduces (81), (82) to two sub-problems, leading to the auxiliary equation for $W$ and the bifurcation equation for $\alpha$. In fact, since we have three parameters $\alpha=\left(\bar{\alpha}, \underline{\alpha}, \alpha_{J}\right)$ and our kernel is essentially one-dimensional, we need only solve for one of the $\alpha$ components, say $\alpha_{J}=\alpha_{J}(\epsilon, \bar{\alpha}, \underline{\alpha})$, and we get a three-parameter family of solutions.

To define the auxiliary equation, refer to $(78),(80)$ and define the operator $\mathcal{F}$ : $I_{\delta} \times B_{\delta} \times \mathcal{K}^{\perp} \rightarrow \Delta$ by

$$
\mathcal{F}(\epsilon, \alpha, W)=\mathcal{F}_{\epsilon, \alpha}[Z+W],
$$

which makes sense for $(\epsilon, \alpha) \in I_{\delta} \times B_{\delta} \subset \mathbb{R}^{4}$ in some neighborhood of the origin. Thus, recalling that $\mathcal{P}$ defined in (69) is projection onto $\mathcal{R}$, we define the auxiliary equation to be

$$
\mathcal{P} \mathcal{F}(\epsilon, \alpha, W)=\mathcal{P} \mathcal{F}_{\epsilon, \alpha}[Z+W]=0
$$

\footnotetext{
${ }^{4}$ Note that the zero mode will not be zero throughout the $y$-evolution $\mathcal{F}_{\epsilon, \alpha}(y)\left[V_{\epsilon, \alpha}\right]$, and so the zero mode cannot be removed from the analysis at the start.
} 
so that

$$
\mathcal{P} \mathcal{F}: I_{\delta} \times B_{\delta} \times \mathcal{K}^{\perp} \rightarrow \mathcal{R}
$$

Based on our strategy above, we wish to solve the auxiliary equation (84) for $W=$ $W(\epsilon, \alpha)$ for some $\epsilon \neq 0$ using an infinite dimensional version of the implicit function theorem. By Lemma 10,

$$
\mathcal{P} \mathcal{F}(0,0, \cdot)=\mathcal{P} \mathcal{F}_{0,0}: \mathcal{K}^{\perp} \rightarrow \mathcal{R}
$$

is linear and invertible, and so we would like to solve (84) uniquely for $W$, for each $(\epsilon, \alpha)$ in a neighborhood of $(0,0)$, by the implicit function theorem. However, this is an infinite dimensional problem with small divisors, so we cannot apply the implicit function theorem directly. To be precise, and to isolate the difficulties of obtaining periodic solutions into the form of a classical implicit function theorem, we make the following definition. In the next section we show that if $\Theta$ is non-resonant, then there exist solutions of the auxiliary equation in this sense under the $N$-Fourier cutoff assumption..$^{5}$

DeFINITION 12. We say that $W$ solves the auxiliary equation in $H^{s}, s \geq 1$, for $\Theta=(\bar{\theta}, \underline{\theta}) \in E_{0}$, if there exists $\delta>0$ and a smooth map

$$
W \equiv W(\epsilon, \alpha): I_{\delta} \times B_{\delta} \rightarrow H^{s},
$$

such that $W(\epsilon, \alpha) \in \mathcal{K}^{\perp} \subset \Delta$ satisfies

$$
W(0,0)=0,
$$

and

$$
\mathcal{P} \mathcal{F}_{\epsilon, \alpha}[Z+W(\epsilon, \alpha)] \equiv 0
$$

for all $(\epsilon, \alpha) \in I_{\delta} \times B_{\delta}$.

Now to define the bifurcation equation, suppose that a solution $W(\epsilon, \alpha)$ satisfying Definition 12 has been found. In this case, we substitute $W(\epsilon, \alpha)$ into (83) and apply the projection $\mathcal{I}-\mathcal{P}$ to get the function

$$
\Phi \equiv \Phi(\epsilon, \alpha): I_{\delta} \times B_{\delta} \rightarrow \mathcal{R}^{\perp}
$$

given by

$$
\begin{aligned}
\Phi(\epsilon, \alpha) & \equiv(\mathcal{I}-\mathcal{P}) \mathcal{F}(\epsilon, \alpha, W(\epsilon, \alpha)) \\
& =(\mathcal{I}-\mathcal{P}) \mathcal{F}_{\epsilon, \alpha}[Z+W(\epsilon, \alpha)],
\end{aligned}
$$

and this yields the bifurcation equation

$$
\Phi(\epsilon, \alpha)=0
$$

\footnotetext{
${ }^{5}$ This is the simplest possible way in which solutions of the auxiliary equation might exist. To prove such an implicit function theorem by Nash-Moser type methods, it may be difficult to rule out nearby resonances, and simpler to prove a theorem valid only for set of positive measure of parameters $\epsilon, \alpha$. This would entail treating the bifurcation and auxiliary equations together at each induction step, but we expect it would not modify the validity of the corresponding Liapunov-Schmidt reduction, c.f. [2].
} 
We now wish to solve the two equations (88) for a function $\alpha=\alpha(\epsilon)$ by the finite dimensional implicit function theorem. Since $\alpha \in \mathbb{R}^{3}$, we have extra parameters and we expect many solutions. In fact, the equations (88) are degenerate and we solve only the 1-mode by the implicit function theorem; as described above, we use conservation to show that the 0 -mode also vanishes. This is accomplished in the following theorem, whose proof is the topic of Section 4.

Theorem 13. Assume that $\Theta=(\bar{\theta}, \underline{\theta}) \in E_{0}$ and there exists a smooth solution $W$ satisfying (85)-(86) of Definition 12. Then there exists $\delta>0$ and a function ${ }^{6}$

$$
\alpha_{J}=\alpha_{J}(\epsilon, \bar{\alpha}, \underline{\alpha}): B_{\delta} \rightarrow \mathbb{R}
$$

such that $\alpha_{J}(0,0,0)=0$, and, if we set

$$
\alpha \equiv\left(\bar{\alpha}, \underline{\alpha}, \alpha_{J}(\epsilon, \bar{\alpha}, \underline{\alpha})\right) \in \mathbb{R}^{3},
$$

then

$$
\Phi(\epsilon, \alpha)=(\mathcal{I}-\mathcal{P}) \mathcal{F}_{\epsilon, \alpha}[Z+W(\epsilon, \alpha)] \equiv 0
$$

for all $(\epsilon, \bar{\alpha}, \underline{\alpha}) \in B_{\delta}$.

Theorem 13 reduces existence of time periodic solutions of the compressible Euler equations to existence of solutions $W$ of the auxiliary equation satisfying Definition 12 , as recorded in the following corollary:

TheOREM 14. Assume that $\Theta=(\bar{\theta}, \underline{\theta}) \in E_{0}$ and there exists a smooth solution $W$ satisfying (85)-(86) of Definition 12. Then there are smooth functions $\alpha_{J}(\epsilon, \bar{\alpha}, \underline{\alpha})$ and $V_{\epsilon, \bar{\alpha}, \underline{\alpha}} \in \Delta$,

$$
V_{\epsilon, \bar{\alpha}, \underline{\alpha}}:[0,2 \pi) \rightarrow \mathbb{R}^{2},
$$

such that

$$
V_{0,0,0}=Z=\mathcal{T}_{1}^{*} \mathbf{q} \in \mathcal{K}, \quad \alpha_{J}(0,0,0)=0,
$$

and for some positive constant $\delta>0$,

$$
\left(\mathcal{N}_{\alpha}-\mathcal{I}\right)\left[\mathbf{1}+\epsilon Z+\epsilon W_{\epsilon, \alpha}\right]=0
$$

for all $(\epsilon, \bar{\alpha}, \underline{\alpha}) \in B_{\delta}$, where $\alpha$ is given by (90). In other words, the function $\mathbf{1}+\epsilon Z+$ $\epsilon W_{\epsilon, \alpha}$ is the Cauchy data (for evolution in space) for periodic solutions of compressible Euler.

Proof. By Definition 12, $W=W(\epsilon, \alpha)$ satisfies (84) for all $(\epsilon, \alpha)$, and by Theorem 13 , if $\alpha$ is given by (90), then the bifurcation equation (88) holds. Adding (84) and (87), it follows that

$$
V_{\epsilon, \bar{\alpha}, \underline{\alpha}} \equiv Z+W(\epsilon, \alpha(\epsilon, \bar{\alpha}, \underline{\alpha})) \in \Delta
$$

satisfies (79), namely

$$
\mathcal{F}_{\epsilon, \alpha(\epsilon, \bar{\alpha}, \underline{\alpha})}[Z+W(\epsilon, \alpha(\epsilon, \bar{\alpha}, \underline{\alpha}))]=0 \text {. }
$$

\footnotetext{
${ }^{6}$ Here we have chosen to give $\alpha_{J}$ as a function of $\bar{\alpha}$ and $\underline{\alpha}$ : we could just as easily choose to solve the bifurcation equation for $\bar{\alpha}$ or $\underline{\alpha}$ instead, see (112). Our choice of $\alpha_{J}$ is convenient as it allows us to preserve the symmetry $\bar{\alpha}=\underline{\alpha}$ if desired.
} 
Using (77), (78), this in turn implies

$$
\mathcal{N}_{\alpha(\epsilon, \bar{\alpha}, \underline{\alpha})}\left[\mathbf{1}+\epsilon Z+\epsilon W_{\epsilon, \alpha(\epsilon, \bar{\alpha}, \underline{\alpha})}\right]=\mathbf{1}+\epsilon Z+\epsilon W_{\epsilon, \alpha(\epsilon, \bar{\alpha}, \underline{\alpha})},
$$

this being a three parameter $(\epsilon, \bar{\alpha}, \underline{\alpha})$-family of periodic solutions to (20) that bifurcate from the linearized solution $Z=\overline{\mathcal{T}}_{1}^{*} \mathbf{q}$.

Note that for the family of solutions $V_{\epsilon, \bar{\alpha}, \underline{\alpha}}$, the parameters $(\epsilon, \bar{\alpha}, \underline{\alpha})$ can be freely chosen in the neighborhood $B_{\delta}$ of zero, which implies the existence of periodic solutions with full symmetry $(\bar{\alpha}=\underline{\alpha})$ and arbitrary asymmetry $(\bar{\alpha} \neq \underline{\alpha})$, under the assumption that a solution $W$ of the auxiliary equation exists at a single value of $\Theta$. Moreover, if we choose $(\bar{\alpha}, \underline{\alpha})$ to be $O(|\epsilon|)$, then $W_{\epsilon, \alpha}=O(|\epsilon|)$, so that $\epsilon W_{\epsilon, \alpha(\epsilon, \bar{\alpha}, \underline{\alpha})}=O\left(\epsilon^{2}\right)$, from which it follows that the linearized solutions provide the leading term in our nonlinear periodic solutions, as expected.

It remains to give the proof of Theorem 13, which is the topic of Section 4. That solutions $W$ satisfying Definition 12 exist in the case of the $N$-Fourier cutoff when $\Theta$ is non-resonant is demonstrated in Section 5 .

4. The bifurcation equation. In this section we give the proof of Theorem 13. We assume a fixed (non-symmetric) value of $\Theta=(\bar{\theta}, \underline{\theta}) \in E_{0}$, and assume there exists a corresponding smooth solution $W$ satisfying (85)-(86) of Definition 12 . That is, assume $W$ is a smooth (at least $H^{1}$ ) solution of the infinite-dimensional auxiliary equation (84),

$$
W \equiv W_{\epsilon, \alpha} \equiv W(\epsilon, \alpha), \quad \text { with } \quad \mathcal{P} \mathcal{F}(\epsilon, \alpha, W)=0, \quad W_{0,0}=0 .
$$

We wish to prove that the bifurcation equation (88) can be solved for a function $\alpha_{J}(\epsilon, \bar{\alpha}, \underline{\alpha})$ in a neighborhood of $(0,0,0)$. That such a solution always exists is the claim of Theorem 13, and it is the purpose of this section to prove it.

The bifurcation equation (88) is given by

$$
\Phi(\epsilon, \alpha) \equiv(\mathcal{I}-\mathcal{P}) \mathcal{F}(\epsilon, \alpha, W(\epsilon, \alpha))=0,
$$

where

$$
\Phi: I_{\delta} \times B_{\delta} \subset \mathbb{R}^{4} \rightarrow \mathcal{R}^{\perp},
$$

and according to (68), $\mathcal{R}^{\perp}$ is two-dimensional, consisting of the direct sum

$$
\mathcal{R}^{\perp}=\Delta_{0} \oplus \mathcal{K}_{1}^{\perp}
$$

of the 0-mode

$$
\Delta_{0}=\operatorname{Span}\left\{\mathcal{T}_{0}^{*} \mathbf{1}\right\}
$$

and the one-dimensional kernel in the 1-mode

$$
\mathcal{K}_{1}^{\perp} \equiv \operatorname{Span}\left\{\mathcal{T}_{1}^{*} \mathbf{q}^{\perp}\right\} .
$$

The main difficulty here is that for $\epsilon \neq 0$ the evolution is nonlinear, so the values of $W(\epsilon, \alpha)$, which are unknown, influence the values of $\Phi(\epsilon, \alpha)$.

To start, let $\varphi=\left(\varphi_{0}, \varphi_{1}\right)$, where we define the real valued functions $\varphi_{0}$ and $\varphi_{1}$ as the projections of $\Phi(\epsilon, \alpha)$ onto the zero-mode $\Delta_{0}$ and 1-mode $\mathcal{K}_{1}^{\perp}$, respectively, using the identity

$$
\Phi(\epsilon, \alpha) \equiv \varphi_{0}(\epsilon, \alpha) \mathcal{T}_{0}^{*} \mathbf{1}+\varphi_{1}(\epsilon, \alpha) \mathcal{T}_{1}^{*} \mathbf{q}^{\perp}
$$


Alternatively, we may rewrite the definitions as

$$
\varphi_{1}(\epsilon, \alpha)=\left\langle\mathcal{T}_{1}^{*} \mathbf{q}^{\perp}, \Phi(\epsilon, \alpha)\right\rangle,
$$

and

$$
\varphi_{0}(\epsilon, \alpha)=\left\langle\mathcal{T}_{0}^{*} 1, \Phi(\epsilon, \alpha)\right\rangle .
$$

Our strategy for the proof of Theorem 13 is to apply the implicit function theorem to $\varphi_{1}$ to prove that the function $\varphi_{1}(\epsilon, \alpha)$ vanishes along a surface $\alpha=\alpha(\epsilon, \bar{\alpha}, \underline{\alpha})$ in some neighborhood $B_{\delta}$ of zero, $(\epsilon, \bar{\alpha}, \underline{\alpha}) \in B_{\delta} \subset \mathbb{R}^{3}$. We then use a conserved moment in $w$, an integral constant of motion for the nonlinear problem derivable from the conservation form of the equations, to prove that on the surface $\alpha=\alpha(\epsilon, \bar{\alpha}, \underline{\alpha})$, $\varphi_{0}(\epsilon, \alpha)$ also vanishes. We can then conclude from (93) that $\Phi(\epsilon, \alpha)=0$ on the surface $\alpha=\alpha(\epsilon, \bar{\alpha}, \underline{\alpha}),(\epsilon, \bar{\alpha}, \underline{\alpha}) \in B_{\delta}$, the result we desire.

More precisely, to obtain $\alpha=\alpha(\epsilon, \bar{\alpha}, \underline{\alpha})$, we apply the implicit function theorem to $\varphi_{1}$ in variable $\alpha_{J}$, which means that we need to verify the zero condition

$$
\varphi_{1}(0,0)=0
$$

and the derivative condition

$$
\left.\frac{\partial \varphi_{1}}{\partial \alpha_{J}}\right|_{(0,0)} \neq 0
$$

Together these imply the existence of $\delta>0$ and function

$$
\alpha_{J}=\alpha_{J}(\epsilon, \bar{\alpha}, \underline{\alpha}): B_{\delta} \rightarrow \mathbb{R}
$$

such that

$$
\varphi_{1}(\epsilon, \alpha(\epsilon, \bar{\alpha}, \underline{\alpha}))=0
$$

where we define

$$
\alpha(\epsilon, \bar{\alpha}, \underline{\alpha}) \equiv\left(\bar{\alpha}, \underline{\alpha}, \alpha_{J}(\epsilon, \bar{\alpha}, \underline{\alpha})\right) .
$$

In the final step we use conservation of mass to prove that $\varphi_{0}$ vanishes when (96) holds. Thus we have the

Proof of Theorem 13. The proof hinges on three lemmas which are proved below. In Lemma 19, we first show that

$$
\varphi_{0}(0,0)=\varphi_{1}(0,0)=0 .
$$

Next, in Lemma 20, we show that for any $\Theta \in E_{0}$, the derivatives

$$
\frac{\partial \varphi_{1}}{\partial \bar{\alpha}}, \quad \frac{\partial \varphi_{1}}{\partial \underline{\alpha}} \text { and } \frac{\partial \varphi_{1}}{\partial \alpha_{J}}
$$

are all nonzero. It then follows by the implicit function theorem that we can solve $\varphi_{1}=0$ for $\alpha_{J}$ (or any parameter): that is, there is a $\delta>0$ and a function (89) such that if $\alpha$ is given by (90), then

$$
\varphi_{1}(\epsilon, \alpha)=0 \quad \text { for all } \quad(\epsilon, \bar{\alpha}, \underline{\alpha}) \in B_{\delta} .
$$

Finally, in Lemma 21 below, we prove that, since $W_{\epsilon, \alpha}$ solves the auxiliary equation, if $\varphi_{1}=0$ in $B_{\delta}$, then $\varphi_{0}(\epsilon, \alpha)=0$ there as well. We conclude that $\Phi(\epsilon, \alpha)=0$ on the surface $\alpha_{J}=\alpha_{J}(\epsilon, \bar{\alpha}, \underline{\alpha})$ for $(\epsilon, \bar{\alpha}, \underline{\alpha}) \in B_{\delta}$. Thus the proof is complete once we state and prove Lemmas 19, 20 and 21 . 
4.1. Expansion of the solution. In order to prove the lemmas, we need explicit expressions for $\varphi_{0}$ and $\varphi_{1}$, at least for $\epsilon=0$. To get these we need to know the leading order behavior of $W(\epsilon, \alpha)$.

LEMma 15. The solution $W(\epsilon, \alpha)$ of the auxiliary equation (86) satisfies

$$
W(\epsilon, \alpha)=c_{W}(\alpha) \mathcal{T}_{1}^{*} \mathbf{q}^{\perp}+O(\epsilon)
$$

where $c_{W}(\alpha)$ is given by

$$
c_{W}(\alpha)=\frac{1-\mathbf{q} \cdot M_{\alpha} \mathbf{q}}{\mathbf{q} \cdot M_{\alpha} \mathbf{q}^{\perp}},
$$

satisfies $c_{W}(\alpha)=O(\alpha)$, and $M_{\alpha}$ is given by (73), namely

$$
M_{\alpha}=-D_{\alpha}^{-1} R(\underline{\theta}+\underline{\alpha}) D_{\alpha} R(\bar{\theta}+\bar{\alpha}) .
$$

Proof. Since $W(\epsilon, \alpha)$ is the unique solution of $\mathcal{P F}_{\epsilon, \alpha}[Z+W]=0$ in $I_{\delta} \times B_{\delta}$, it follows that for $\epsilon=0, W(0, \alpha)$ solves the linearized problem

$$
\mathcal{P} \mathcal{M}_{\alpha}[Z+W]=\mathcal{P}[Z+W]
$$

where $\mathcal{P}$ is projection onto $\mathcal{R}$. Since the linearized problem preserves modes and $\mathcal{P}$ is the identity on each $\Delta_{n}, n \geq 2$, uniqueness of the solution implies that

$$
W(0, \alpha) \in \mathcal{K}^{\perp} \cap \Delta_{1}=\mathcal{K}_{1}^{\perp}=\operatorname{Span}\left\{\mathcal{T}_{1}^{*} \mathbf{q}^{\perp}\right\}
$$

It follows that $W(\epsilon, \alpha)$ is of the form (97), and we get $c_{W}(\alpha)$ by considering the 1-mode projection of (99): as in Theorem 2, using (72), (73), we have

$$
\mathcal{M}_{\alpha} \mathcal{T}_{1}^{*}=\mathcal{T}_{1}^{*} M_{\alpha}
$$

and $\mathcal{P}$ projects 1 -modes onto $\operatorname{Span}\{\mathbf{q}\}$, so that (99) becomes

$$
\mathbf{q} \cdot M_{\alpha}\left(\mathbf{q}+c_{W}(\alpha) \mathbf{q}^{\perp}\right)=\mathbf{q} \cdot\left(\mathbf{q}+c_{W}(\alpha) \mathbf{q}^{\perp}\right)=1,
$$

and solving for $c_{W}(\alpha)$ yields (98). Finally, when $\alpha=0$, we have $M_{\alpha}=M_{1}$, so that $M_{\alpha} \mathbf{q}=\mathbf{q}$, while $\mathbf{q} \cdot M_{1} \mathbf{q}^{\perp} \neq 0$, so that $c_{W}(0)=0$ and thus also $c_{W}(\alpha)=O(\alpha)$.

We will need the derivatives of $c_{W}(\alpha)$ evaluated at $\alpha=0$ :

Corollary 16. For $x=\bar{\alpha}, \underline{\alpha}$ or $\alpha_{J}$, we have

$$
\left.\frac{\partial}{\partial x} c_{W}(\alpha)\right|_{\alpha=0}=\left.\frac{-1}{\tau_{0}} \mathbf{q} \cdot \frac{\partial}{\partial x} M_{\alpha}\right|_{\alpha=0} \mathbf{q},
$$

where $\tau_{0}$ is given by (60).

Proof. This follows immediately by differentiating (100) and using the fact that $c_{W}(0)=0$, together with $(63)$.

In order to get the leading behavior of $\Phi(\epsilon, \alpha)$ given in (92), we need a description of $\mathcal{F}(\epsilon, \alpha, W(\epsilon, \alpha))$, which requires us to evaluate (to leading order) the nonlinear evolution

$$
\mathcal{N}_{\alpha}[\mathbf{1}+\epsilon(Z+W)]
$$


Thus let

$$
U_{0}=\mathbf{1}+\epsilon Z+\epsilon W(\epsilon, \alpha) \equiv U_{\epsilon, \alpha}(0)
$$

be "initial data" which we evolve by the nonlinear operator $\mathcal{N}_{\alpha}$, giving the corresponding solution $U_{\epsilon, \alpha}$, and using the notation (75), we set

$$
U_{\epsilon, \alpha}(y)=\mathcal{N}_{\alpha}(y)\left[U_{0}\right] \in \Delta
$$

which has values

$$
U_{\epsilon, \alpha}(y, t) \equiv \mathbf{1}+\epsilon V(y, t),
$$

where $V(y, t)$ is the rescaled solution of the PDE. In coordinates, we write

$$
V(y)=\left[\begin{array}{l}
w(y) \\
v(y)
\end{array}\right]=\sum_{n=0}^{\infty} \mathcal{T}_{n}^{*}\left[\begin{array}{c}
w_{n}(y) \\
v_{n}(y)
\end{array}\right]
$$

so that, if we write

$$
U_{\epsilon, \alpha}(y, t)=\left[\begin{array}{c}
w_{\epsilon, \alpha}(y, t) \\
v_{\epsilon, \alpha}(y, t)
\end{array}\right]
$$

then

$$
\begin{aligned}
& w_{\epsilon, \alpha}(y, t)=1+\epsilon \sum_{n=0}^{\infty} w_{n}(y) \cos n t \\
& v_{\epsilon, \alpha}(y, t)=\epsilon \sum_{n=0}^{\infty} v_{n}(y) \sin n t .
\end{aligned}
$$

For $0<y<\bar{\theta}+\bar{\alpha}$ and $0<y-\bar{\theta}-\bar{\alpha}<\underline{\theta}+\underline{\alpha}$, the functions $w_{\epsilon, \alpha}(y, t)$ and $v_{\epsilon, \alpha}(y, t)$ solve the PDEs (20). Now $\mathcal{N}_{\alpha}=\mathcal{M}_{\alpha}$ to leading order in $\epsilon$, which implies the following theorem:

LEMma 17. The leading order evolution for $V=Z+W$ in the modes $n \geq 1$ determined by (102) is given in the intervals

$$
y \in(0, \bar{\theta}+\bar{\alpha}) \quad \text { and } \quad y \in(\bar{\theta}+\bar{\alpha}, \bar{\theta}+\bar{\alpha}+\underline{\theta}+\underline{\alpha}),
$$

by the equations

$$
\begin{aligned}
& w_{n}^{\prime}+n v_{n}=O(\epsilon), \\
& v_{n}^{\prime}-n w_{n}=O(\epsilon) .
\end{aligned}
$$

Proof. Using (103) in the equations (20) and letting $u^{\prime}$ denote differentiation of $u$ with respect to $y$, we get

$$
\begin{aligned}
& \sum_{n \geq 0} w_{n}^{\prime} \cos n t+\sum_{n \geq 1} n v_{n} \cos n t=O(\epsilon), \\
& \sum_{n \geq 0} v_{n}^{\prime} \sin n t-\sum_{n \geq 1} n w_{n} \sin n t=O(\epsilon) .
\end{aligned}
$$


Separating modes and taking $n \geq 1$ directly gives (104).

The next lemma provides a formula for $\varphi_{1}(0, \alpha)$.

LEMma 18. At $\epsilon=0$, we have the identity

$$
\begin{aligned}
\varphi_{1}(0, \alpha) & =\mathbf{q}^{\perp} \cdot\left(M_{\alpha}-I\right)\left[\mathbf{q}+c_{W}(\alpha) \mathbf{q}^{\perp}\right] \\
& =\mathbf{q}^{\perp} \cdot M_{\alpha} \mathbf{q}+c_{W}(\alpha) \mathbf{q}^{\perp} \cdot\left(M_{\alpha}-I\right) \mathbf{q}^{\perp},
\end{aligned}
$$

where $c_{W}(\alpha)$ is given in (98).

Proof. From the definitions (88), (78) and (77), and (102), we have that

$$
\Phi(\epsilon, \alpha)=V(\bar{\theta}+\bar{\alpha}+\underline{\theta}+\underline{\alpha})-V(0),
$$

where

$$
V(0)=Z+W(\epsilon, \alpha) \quad \text { and } \quad Z=\mathcal{T}_{1}^{*} \mathbf{q} .
$$

It follows from (94), using the inner product (57), that

$$
\varphi_{1}(\epsilon, \alpha)=\mathbf{q}^{\perp} \cdot\left\{\left[\begin{array}{c}
w_{1}(\bar{\theta}+\bar{\alpha}+\underline{\theta}+\underline{\alpha}) \\
v_{1}(\bar{\theta}+\bar{\alpha}+\underline{\theta}+\underline{\alpha})
\end{array}\right]-\left[\begin{array}{c}
w_{1}(0) \\
v_{1}(0)
\end{array}\right]\right\} .
$$

Now set $\epsilon=0$ in (107). Then (104) becomes the linear system

$$
V_{1}^{\prime}=P V_{1}
$$

where the matrix $P$ is defined by

$$
P \equiv\left(\begin{array}{cc}
0 & -1 \\
1 & 0
\end{array}\right)
$$

Note that $P=R(\pi / 2)$ satisfies

$$
e^{x P}=R(x),
$$

so that evolution through an interval of length $x$ is simply rotation by $x$, as seen in our earlier analysis of the linear equations. Putting in the jumps and shift, it follows that

$$
V_{1}(\bar{\theta}+\bar{\alpha}+\underline{\theta}+\underline{\alpha})=M_{\alpha} V_{1}(0)
$$

where

$$
V_{1}(y)=\left[\begin{array}{c}
\left.w_{1}(y)\right|_{\epsilon=0} \\
\left.v_{1}(y)\right|_{\epsilon=0}
\end{array}\right] \equiv\left[\begin{array}{c}
w_{\alpha}(y) \\
v_{\alpha}(y)
\end{array}\right]
$$

gives the the solution for $\epsilon=0$ for $y$ throughout the interval $(0, \bar{\theta}+\bar{\alpha}+\underline{\theta}+\underline{\alpha})$. Finally, using (80) and (97), we get

$$
V_{1}(0)=\mathbf{q}+c_{W}(\alpha) \mathbf{q}^{\perp},
$$

and substituting into (110) and (107) gives (105).

The next lemma records the vanishing of $\varphi_{1}$ and $\varphi_{0}$ at the origin.

LEMMA 19. The functions $\varphi_{1}$ and $\varphi_{0}$ vanish at the origin,

$$
\varphi_{1}(0,0)=\varphi_{0}(0,0)=0 .
$$

Proof. That $\varphi_{0}(0,0)=0$ follows from the fact that the evolution part of the linearized operator $\mathcal{M}$ is in conservation form. On the other hand, by (73) and (41),

$$
\left.M_{\alpha}\right|_{\alpha=0}=-D^{-1} R(\underline{\theta}) D R(\bar{\theta})=M_{1},
$$

and, since $c_{W}(0)=0,(105)$ yields $\varphi_{1}(0,0)=\mathbf{q}^{\perp} \cdot M_{1} \mathbf{q}=0$. 
4.2. Derivative calculations. To apply the implicit function theorem to solve $\varphi_{1}=0$, we must show that the derivative with respect to $\alpha_{J}$ is non-zero at the origin,

$$
\left.\frac{d \varphi_{1}}{d \alpha_{J}}\right|_{\epsilon, \alpha=0} \neq 0
$$

More generally, we calculate the full gradient of $\varphi_{1}$ with respect to $\alpha$ at the origin.

LEMma 20. We have the formulas

$$
\frac{\partial \varphi_{1}}{\partial \bar{\alpha}}=1, \quad \frac{\partial \varphi_{1}}{\partial \underline{\alpha}}=\frac{\sin \bar{\theta}}{\sin \underline{\theta}}, \quad \text { and } \quad \frac{\partial \varphi_{1}}{\partial \alpha_{J}}=\frac{\sin \bar{\theta}}{J},
$$

all derivatives being evaluated at the origin $(\epsilon, \alpha)=0 \in \mathbb{R}^{4}$. In particular, none of these derivatives vanish if $\Theta \in E_{0}$.

Proof. Referring to (105), we first note that since $c_{W}(\alpha)=O(\alpha)$ and since

$$
\left.\operatorname{Range}\left(M_{\alpha}-I\right)\right|_{\alpha=0}=\operatorname{Range}\left(M_{1}-I\right)=\operatorname{Span}\{\mathbf{q}\},
$$

we have for $x=\bar{\alpha}, \underline{\alpha}$ or $\alpha_{J}$,

$$
\left.\frac{\partial}{\partial x}\left\{c_{W}(\alpha) \mathbf{q}^{\perp} \cdot\left(M_{\alpha}-I\right) \mathbf{q}^{\perp}\right\}\right|_{\alpha=0}=0,
$$

which implies

$$
\left.\frac{\partial}{\partial x} \varphi_{1}(0, \alpha)\right|_{\alpha=0}=\left.\mathbf{q}^{\perp} \cdot \frac{\partial M_{\alpha}}{\partial x}\right|_{\alpha=0} \mathbf{q} .
$$

We have by (73),

$$
M_{\alpha}=-D_{\alpha}^{-1} R(\underline{\theta}+\underline{\alpha}) D_{\alpha} R(\bar{\theta}+\bar{\alpha}),
$$

and also, using (108),

$$
\frac{d}{d x} R(x)=\frac{d}{d x} e^{x P}=P e^{x P}=P R(x)=R(x+\pi / 2) .
$$

It follows from (114) that when $\alpha=0$,

$$
\frac{\partial \varphi_{1}}{\partial \bar{\alpha}}=-\mathbf{q}^{\perp} \cdot D^{-1} R(\underline{\theta}) D R(\bar{\theta}) P \mathbf{q},
$$

and

$$
\frac{\partial \varphi_{1}}{\partial \underline{\alpha}}=-\mathbf{q}^{\perp} \cdot D^{-1} P R(\underline{\theta}) D R(\bar{\theta}) \mathbf{q} .
$$

Similarly,

$$
\begin{aligned}
\frac{\partial \varphi_{1}}{\partial \alpha_{J}}=- & \mathbf{q}^{\perp} \cdot\left(\begin{array}{cc}
0 & 0 \\
0 & \frac{-1}{J^{2}}
\end{array}\right) R(\underline{\theta}) D R(\bar{\theta}) \mathbf{q} \\
& -\mathbf{q}^{\perp} \cdot D^{-1} R(\underline{\theta})\left(\begin{array}{cc}
0 & 0 \\
0 & 1
\end{array}\right) R(\bar{\theta}) \mathbf{q} .
\end{aligned}
$$


For (116), by (113), we have for any $\mathbf{p} \in \mathbb{R}^{2}$,

$$
\mathbf{q}^{\perp} \cdot\left(M_{1}-I\right) \mathbf{p}=0, \quad \text { so that } \mathbf{q}^{\perp} \cdot M_{1} \mathbf{p}=\mathbf{q}^{\perp} \cdot \mathbf{p},
$$

so that

$$
\frac{\partial \varphi_{1}}{\partial \bar{\alpha}}=\mathbf{q}^{\perp} \cdot P \mathbf{q}=\mathbf{q}^{\perp} \cdot \mathbf{q}^{\perp}=1 .
$$

Next, to evaluate (117), write

$$
D^{-1} P=D^{-1} P D D^{-1}
$$

and recall that $\left.M_{\alpha}\right|_{0} \mathbf{q}=M_{1} \mathbf{q}=\mathbf{q}$, to get

$$
\begin{aligned}
\frac{\partial \varphi_{1}}{\partial \underline{\alpha}} & =-\mathbf{q}^{\perp} \cdot\left(D^{-1} P D\right)\left(-M_{1}\right) \mathbf{q} \\
& =\mathbf{q}^{\perp} \cdot D^{-1} P D \mathbf{q} .
\end{aligned}
$$

Now

$$
\mathbf{q}=\left(\begin{array}{c}
\cos \bar{\theta} / 2 \\
-\sin \bar{\theta} / 2
\end{array}\right), \quad \mathbf{q}^{\perp}=\left(\begin{array}{c}
\sin \bar{\theta} / 2 \\
\cos \bar{\theta} / 2
\end{array}\right) \quad \text { and } \quad D=\left(\begin{array}{cc}
1 & 0 \\
0 & J
\end{array}\right)
$$

so continuing from (119) we have

$$
\begin{aligned}
\frac{\partial \varphi_{1}}{\partial \underline{\alpha}} & =\left(\begin{array}{ll}
\sin \bar{\theta} / 2 & \frac{1}{J} \cos \bar{\theta} / 2
\end{array}\right)\left(\begin{array}{cc}
0 & -1 \\
1 & 0
\end{array}\right)\left(\begin{array}{c}
\cos \bar{\theta} / 2 \\
-J \sin \bar{\theta} / 2
\end{array}\right) \\
& =\left(\begin{array}{ll}
\frac{1}{J} \cos \bar{\theta} / 2 & -\sin \bar{\theta} / 2
\end{array}\right)\left(\begin{array}{c}
\cos \bar{\theta} / 2 \\
-J \sin \bar{\theta} / 2
\end{array}\right), \\
& =\frac{1}{J} \cos ^{2} \bar{\theta} / 2+J \sin ^{2} \bar{\theta} / 2 .
\end{aligned}
$$

Now using (49) to eliminate $J$, we get

$$
\begin{aligned}
\frac{\partial \varphi_{1}}{\partial \underline{\alpha}} & =\cos \bar{\theta} / 2 \sin \bar{\theta} / 2\left(\frac{\sin \underline{\theta} / 2}{\cos \underline{\theta} / 2}+\frac{\cos \underline{\theta} / 2}{\sin \underline{\theta} / 2}\right) \\
& =\frac{\cos \bar{\theta} / 2 \sin \bar{\theta} / 2}{\cos \underline{\theta} / 2 \sin \underline{\theta} / 2}=\frac{\sin \bar{\theta}}{\sin \underline{\theta}},
\end{aligned}
$$

by the double angle formula.

Finally, we use $M_{1} \mathbf{q}=\mathbf{q}$ to rewrite (118) as

$$
\begin{aligned}
\frac{\partial \varphi_{1}}{\partial \alpha_{J}}=\frac{-1}{J} & \mathbf{q}^{\perp} \cdot\left(\begin{array}{cc}
0 & 0 \\
0 & 1
\end{array}\right) \mathbf{q} \\
& -\mathbf{q}^{\perp} \cdot D^{-1} R(\underline{\theta})\left(\begin{array}{ll}
0 & 0 \\
0 & 1
\end{array}\right)\left[\begin{array}{c}
\cos \bar{\theta} / 2 \\
\sin \bar{\theta} / 2
\end{array}\right],
\end{aligned}
$$

which simplifies to

$$
\begin{aligned}
\frac{\partial \varphi_{1}}{\partial \alpha_{J}}=\frac{1}{J} & \sin \bar{\theta} / 2 \cos \bar{\theta} / 2 \\
& +\sin ^{2} \bar{\theta} / 2 \sin \underline{\theta}-\frac{1}{J} \sin \bar{\theta} / 2 \cos \bar{\theta} / 2 \cos \underline{\theta} .
\end{aligned}
$$


Now, using the double angle formulas and (49), we calculate

$$
\begin{aligned}
\frac{\partial \varphi_{1}}{\partial \alpha_{J}} & =\frac{\sin \frac{\bar{\theta}}{2} \cos \frac{\bar{\theta}}{2}}{J}\left(1-\cos \underline{\theta}+\frac{J \sin \frac{\bar{\theta}}{2}}{\cos \frac{\bar{\theta}}{2}} \sin \underline{\theta}\right) \\
& =\frac{\sin \bar{\theta}}{2 J}\left(1-\left(\cos ^{2} \frac{\theta}{\overline{2}}-\sin ^{2} \frac{\theta}{2}\right)+\frac{\cos \frac{\theta}{2}}{\sin \frac{\theta}{2}} 2 \sin \frac{\theta}{2} \cos \frac{\theta}{2}\right),
\end{aligned}
$$

which simplifies to give the last relation in (112).

4.3. Evolution of the moment. In light of Lemmas 19 and 20, we can apply the implicit function theorem to solve $\varphi_{1}=0$. The proof of Theorem 13 is thus complete once we prove that $\varphi_{0}$ vanishes when $\varphi_{1}$ does. Recall that we are assuming that $W_{\epsilon, \alpha}$ solves the auxiliary equation.

THEOREM 21. Assume that a function $\alpha_{J}=\alpha_{J}(\epsilon, \bar{\alpha}, \underline{\alpha}): B_{\delta} \rightarrow \mathbb{R}$ is given for which

$$
\varphi_{1}(\epsilon, \alpha)=0 \quad \text { for all } \quad(\epsilon, \bar{\alpha}, \underline{\alpha}) \in B_{\delta},
$$

Then we also have

$$
\varphi_{0}(\epsilon, \alpha)=0 \quad \text { for all } \quad(\epsilon, \bar{\alpha}, \underline{\alpha}) \in B_{\delta} \text {. }
$$

Proof. Assume that $(\epsilon, \bar{\alpha}, \underline{\alpha}) \in B_{\delta}$, set

$$
\alpha \equiv\left(\bar{\alpha}, \underline{\alpha}, \alpha_{J}(\epsilon, \bar{\alpha}, \underline{\alpha})\right) \in \mathbb{R}^{3},
$$

as in (90), and define

$$
V_{0} \equiv Z+W_{\epsilon, \alpha} \in \Delta \text {. }
$$

Then, using (86), (87) and (120), we get

$$
\begin{aligned}
\mathcal{F}_{\epsilon, \alpha}\left[V_{0}\right] & =\mathcal{P} \mathcal{F}_{\epsilon, \alpha}\left[V_{0}\right]+\Phi(\epsilon, \alpha) \\
& =\varphi_{0}(\epsilon, \alpha) \mathcal{T}_{0}^{*} \mathbf{1}=\varphi_{0}(\epsilon, \alpha) \mathbf{1},
\end{aligned}
$$

which, by (78) and (77), implies that

$$
\mathcal{N}_{\alpha}\left[\mathbf{1}+\epsilon V_{0}\right]=\left(1+\epsilon \varphi_{0}(\epsilon, \alpha)\right) \mathbf{1}+\epsilon V_{0},
$$

and we note that $V_{0}$ has no component in $\Delta_{0}$. Now define

$$
\dot{U}(t)=(\dot{w}(t), \dot{v}(t)) \equiv \mathbf{1}+\epsilon V_{0}(t)
$$

and set

$$
U^{\#}=\left(w^{\#}(t), v^{\#}(t)\right) \equiv \mathcal{N}_{\alpha}[\dot{U}],
$$

so that $U^{\#}$ is the (shifted) spatial evolution (with jumps) of $\dot{U}$ through the period. Then by (122), we have

$$
\operatorname{Proj}_{\Delta_{n}} U^{\#}=\operatorname{Proj}_{\Delta_{n}} \dot{U} \quad \text { for all } \quad n \geq 1
$$


and it follows that

$$
w^{\#}(t)=\dot{w}(t)+w_{0}, \quad \text { and } \quad v^{\#}(t)=\dot{v}(t)
$$

with $w_{0}=\epsilon \varphi_{0}(\epsilon, \alpha)$, a constant with respect to the nonlinear evolution. Thus to verify (121), it remains only to show that $w_{0}=0$.

According to (72),

$$
\mathcal{N}_{\alpha} \equiv \mathcal{S} \cdot \mathcal{J}_{\alpha}^{-1} \cdot \mathcal{E}(\underline{\theta}+\underline{\alpha}) \cdot \mathcal{J}_{\alpha} \cdot \mathcal{E}(\bar{\theta}+\bar{\alpha}),
$$

and so $U^{\#}$ is obtained from $\dot{U}$ by two nonlinear evolutions $\mathcal{E}(\bar{\theta}+\bar{\alpha})$ and $\mathcal{E}(\underline{\theta}+\underline{\alpha})$, two linear jumps $\mathcal{J}_{\alpha}, \mathcal{J}_{\alpha}^{-1}$, and a linear half period shift $\mathcal{S}$. By $(25), w$ is constant across entropy jumps, while the two nonlinear evolutions correspond to evolution by the nonlinear non-conservative system (20). This is equivalent, by change of variables, to evolution by the $p$-system

$$
\begin{aligned}
& \tau_{t}-u_{x}=0, \\
& u_{t}+p_{x}=0,
\end{aligned}
$$

on each entropy level, since the entropy $S$ is constant on each evolution. Since the $p$-system is in conservation form, we integrate in time to get

$$
\frac{d}{d x} \int_{0}^{2 \pi} p(x, t) d t=0
$$

so this integral is constant in $x$ on each entropy level. Note that this is not the usual conserved integral as we have interchanged the roles of $x$ and $t$ for the evolution. Now, by (9), (19) we have

$$
p=K_{p} m^{2}\left(z_{0} w\right)^{\frac{2 \gamma}{\gamma-1}}
$$

so it follows directly using (18) that

$$
\frac{d}{d y} \int_{0}^{2 \pi} w(y, t)^{\frac{2 \gamma}{\gamma-1}} d t=0
$$

separately on each entropy level. Therefore, since $w$ is preserved by the jumps and the moment is preserved under a time-shift, this integral is constant throughout the entire evolution. We thus conclude that

$$
\int_{0}^{2 \pi}\{\dot{w}(t)\}^{\frac{2 \gamma}{\gamma-1}} d t=\int_{0}^{2 \pi}\left\{w^{\#}(t)\right\}^{\frac{2 \gamma}{\gamma-1}} d t
$$

which by (123) implies

$$
\int_{0}^{2 \pi}\left[\left\{w_{0}+\dot{w}(t)\right\}^{\frac{2 \gamma}{\gamma-1}}-\{\dot{w}(t)\}^{\frac{2 \gamma}{\gamma-1}}\right] d t=0 .
$$

Since $\dot{w}(t)>0,(w$, like $\tau$, measures a mass density), it follows that the integrand in (124) has the sign of the constant $w_{0}$ for all values of $t$. But the integral vanishes, so we must have $w_{0}=0$. This completes the proof of the lemma, and the proof of Theorem 13 is complete. 
5. The $N$-Fourier cutoff problem. In this section we show that the assumption of existence of a solution $W$ of the auxiliary equation (84) is valid for non-resonant $\Theta$ if we assume a cutoff of Fourier modes at arbitrarily high modes. In this case the proof of Theorem 14, giving periodic solutions under this assumption, goes through essentially unchanged. We take this as demonstrating that periodic solutions of the compressible Euler equations exist to within an arbitrary Fourier cutoff.

Thus let $N \geq 2$ be any positive natural number, and define the $N$-Fourier cutoff associated with the operators $\mathcal{N}$ and $\mathcal{M}$ by

$$
\mathcal{N}^{N}[V] \equiv \mathbf{1}+\mathcal{P}^{N} \mathcal{N}^{N}[V]
$$

and

$$
\mathcal{M}^{N}[V]=\mathbf{1}+\mathcal{P}^{N} \mathcal{M P}^{N}[V]=\mathbf{1}+\mathcal{M P}^{N}[V]
$$

where $\mathcal{P}^{N}$ denotes orthogonal projection onto the space $\Delta^{N}$ defined by

$$
\Delta^{N} \equiv \mathcal{P}^{N} \Delta=\oplus_{1 \leq n \leq N} \Delta_{n} \subset \Delta
$$

and we restrict ${ }^{7}$

$$
\mathcal{N}^{N}, \mathcal{M}^{N}: \mathbf{1}+\Delta^{N} \rightarrow \mathbf{1}+\Delta^{N}
$$

Since Fourier modes are invariant for the linear operator $\mathcal{M}, \mathcal{M}^{N}$ is just the restriction of $\mathcal{M}$ to the space $\Delta^{N}$, as in Theorem 2. The $N$-Fourier cutoff assumption reduces the infinite dimensional nonlinear and linear eigenvalue problems (1) and (2) to the finite dimensional problems

$$
\left(\mathcal{N}^{N}-\mathcal{I}\right)[V]=0
$$

and

$$
\left(\mathcal{M}^{N}-\mathcal{I}\right)[V]=0
$$

where now $V \in \Delta^{N}$. In particular, by Theorem 2, the kernel of $\mathcal{M}^{N}-\mathcal{I}$ is the projection of the kernel of $\mathcal{M}-\mathcal{I}$ on $\Delta^{N}$. We record this as a lemma:

LEMMA 22. For $N \geq 2$, the kernel and range of $\mathcal{M}^{N}$, are given by

$$
\mathcal{K}^{N} \equiv \mathcal{P}^{N} \mathcal{K} \quad \text { and } \quad \mathcal{R}^{N} \equiv \mathcal{P}^{N} \mathcal{R}
$$

respectively.

The goal of this section is to prove the following theorem:

\footnotetext{
${ }^{7}$ Note that, in addition to removing the high modes $n>N$, we have imposed periodicity of the 0 mode by projecting out the 0 -mode of each operator and then adding back in the non-dimensionalized 0 -mode base state $\mathbf{1}=(1,0)$. The motivation for this is that the proof in Theorem 21 that perodicity of the 0 -mode is a consequence of periodicity of the 1 -mode, requires the conservation law, and the conservation law only holds approximately when high modes are neglected. However, because Theorem 21 demonstrates that the zero mode is periodic when all the Fourier modes are included, the failure of periodicity of the zero mode when high modes are neglected should be on the order of the neglected modes. By this we suggest that (125), (126) are appropriate for an approximate finite dimensional cutoff of the original infinite dimensional problems.
} 
TheOREM 23. Let $N \geq 2$ and let $\Theta=(\bar{\theta}, \underline{\theta}) \in(0, \pi) \times(0, \pi)$ be non-resonant, and let $J$ be given by (49). Then there exists a three parameter family of solutions of (128),

$$
V \equiv V_{\epsilon, \bar{\alpha}, \underline{\alpha}} \in \Delta^{N} \quad \text { such that } \quad \mathcal{N}^{N} V=V,
$$

that bifurcate from the solution $V_{*}=\mathcal{T}_{1}^{*} \mathbf{q} \in \Delta_{1}$ of the linear problem

$$
\mathcal{M}[V]=V, \quad \mathcal{M} \equiv \mathcal{S} \cdot \mathcal{J}^{-1} \cdot \mathcal{L}(\underline{\theta}) \cdot \mathcal{J} \cdot \mathcal{L}(\bar{\theta}) .
$$

As above, the parameter $\epsilon$ measures the strength of the nonlinear perturbation from $\mathcal{M}$, and $(\bar{\alpha}, \underline{\alpha})$ is an arbitrary small perturbation of the period vector $\Theta$, so that $V \equiv V_{\epsilon, \bar{\alpha}, \underline{\alpha}}$ provides solutions of the nonlinear problem (128) for both resonant and non-resonant periods in a neighborhood of a non-resonant $\Theta=(\bar{\theta}, \underline{\theta})$. Since the 1-mode kernel of $\mathcal{M}-\mathcal{I}$ is one dimensional, the result gives, for each non-resonant $\Theta$, the existence of a three parameter family of nonlinear solutions of $\left(\mathcal{N}^{N}-I\right)[V]=0$, the parameters being $(\epsilon, \bar{\alpha}, \underline{\alpha})$, say, that bifurcate from the kernel of the linearized operator $\mathcal{M}-\mathcal{I}$.

To prove the theorem, and paralleling the development in the infinite dimensional case, define

$$
\mathcal{G}_{\epsilon, \alpha}^{N}=\mathcal{P}^{N} \mathcal{G}_{\epsilon, \alpha} \mathcal{P}^{N} \quad \text { and } \quad \mathcal{F}_{\epsilon, \alpha}^{N}=\mathcal{P}^{N} \mathcal{F}_{\epsilon, \alpha} \mathcal{P}^{N}
$$

so that $\mathcal{G}^{N}$ and $\mathcal{F}^{N}$ are finite dimensional operators

$$
\mathcal{G}_{\epsilon, \alpha}^{N}, \mathcal{F}_{\epsilon, \alpha}^{N}: \Delta^{N} \rightarrow \Delta^{N}
$$

where the restriction of $\Delta^{N}$ to Fourier modes $1 \leq n \leq N$ is just $\Delta^{N} \sim \mathbb{R}^{2 N}$, since by (37), the map

$$
\mathcal{T}^{N} \equiv \oplus_{n=1}^{N} \mathcal{T}_{n}^{*}: \mathbb{R}^{2 N} \rightarrow \Delta^{N}
$$

is an isomorphism. In analogy with (84), define the auxiliary equation for the $N$ Fourier cutoff to be

$$
\mathcal{P F}^{N}(\epsilon, \alpha, W)=\mathcal{P} \mathcal{F}_{\epsilon, \alpha}^{N}[Z+W]=0,
$$

so that

$$
\mathcal{P} \mathcal{F}^{N}: I_{\delta} \times B_{\delta} \times \mathcal{P}^{N} \mathcal{K}^{\perp} \rightarrow \mathcal{R}^{N}
$$

The $N$-Fourier cutoff assumption directly implies the following improvements of Lemmas 10 and 11:

Lemma 24. Assume that $N \geq 2$ and that $\Theta$ is nonresonant. Then the operator

$$
\mathcal{M}^{N}-\mathcal{I}^{N}: \mathcal{P}^{N} \mathcal{K}^{\perp} \rightarrow \mathcal{R}^{N}
$$

admits the decomposition

$$
\mathcal{M}^{N}-\mathcal{I}^{N}=\mathcal{P}_{1}\left(\mathcal{M}_{1}-\mathcal{I}\right) \oplus\left\{\oplus_{n=2}^{N}\left(\mathcal{M}_{n}-\mathcal{I}\right)\right\},
$$

and $\mathcal{M}^{N}-\mathcal{I}^{N}: \mathcal{P}^{N} \mathcal{K}^{\perp} \rightarrow \mathcal{R}^{N}$ is bounded and invertible. As in the unbounded case, the 1-mode projection

$$
\mathcal{P}_{1}\left(\mathcal{M}_{1}-\mathcal{I}\right): \mathcal{K}_{1}^{\perp} \rightarrow \mathcal{K}_{1}
$$


is multiplication by $\tau_{0} \neq 0$ as in (70), and we have

$$
\left\|\left[\mathcal{M}^{N}-\mathcal{I}^{N}\right]^{-1}\right\| \leq C(\Theta, N)
$$

where

$$
C(\Theta, N)=O\left(\tau_{0}^{-1}, \max _{2 \leq n \leq N}\left\{\left|(-1)^{n} \lambda_{n}^{ \pm}-1\right|^{-1}\right\}\right)
$$

LEMMA 25. We have

$$
\mathcal{G}_{\epsilon, \alpha}^{N}[V]=\frac{1}{\epsilon}\left\{\mathcal{N}_{\alpha}^{N}[\mathbf{1}+\epsilon V]-\mathbf{1}\right\},
$$

and

$$
\mathcal{F}_{\epsilon, \alpha}^{N}=\mathcal{G}_{\epsilon, \alpha}^{N}-\mathcal{I}^{N}, \quad \text { so that } \quad \mathcal{F}_{\epsilon, \alpha}^{N}[V]=\mathcal{G}_{\epsilon, \alpha}^{N}[V]-V .
$$

The nonlinear operators $\mathcal{G}_{\epsilon, \alpha}^{N}$ and $\mathcal{F}_{\epsilon, \alpha}^{N}$ have the limits

$$
\begin{aligned}
& \lim _{\epsilon \rightarrow 0} \mathcal{F}_{\epsilon, \alpha}^{N}=\mathcal{M}_{\alpha}^{N}-\mathcal{I}^{N} \quad \lim _{\epsilon, \alpha \rightarrow 0} \mathcal{F}_{\epsilon, \alpha}^{N} \equiv \mathcal{G}_{0}^{N}=\mathcal{M}^{N}-\mathcal{I}^{N}, \\
& \lim _{\epsilon \rightarrow 0} \mathcal{G}_{\epsilon, \alpha}^{N}=\mathcal{M}_{\alpha}^{N}, \quad \text { and } \quad \lim _{\epsilon, \alpha \rightarrow 0} \mathcal{G}_{\epsilon, \alpha}^{N}=\mathcal{M}^{N},
\end{aligned}
$$

where convergence is as matrices. Moreover, if

$$
\mathcal{F}_{\epsilon, \alpha}^{N}\left[V_{\epsilon, \alpha}\right]=0
$$

with $\epsilon \neq 0$, then also

$$
\mathcal{N}_{\alpha}^{N}\left[\mathbf{1}+\epsilon V_{\epsilon, \alpha}\right]=\mathbf{1}+\epsilon V_{\epsilon, \alpha} .
$$

Proof. Lemma 24 follows directly by restricting the estimates of Lemma 10 to the Fourier modes $1 \leq n \leq N$. Lemma 25 follows directly from Lemma 11 in light of (125), (126) and (77), (78).

Corollary 26. If $\Theta=(\bar{\theta}, \underline{\theta}) \in(0, \pi) \times(0, \pi)$ is non-resonant, then the auxiliary equation (130) has solutions $W$ in the sense of Definition 12.

Proof. The corollary follows by the implicit function theorem in $\Delta^{N} \sim \mathbb{R}^{2 N}$ in light of the estimate (131) of Lemma 24.

We now have the $N$-Fourier cutoff version of Theorem 13:

THEOREM 27. Assume that $\Theta$ is nonresonant and let $W$ denote the solution of the auxiliary equation (130) satisfying Definition 12. Then there exists $\delta>0$ and a function

$$
\alpha_{J}=\alpha_{J}(\epsilon, \bar{\alpha}, \underline{\alpha}): B_{\delta} \rightarrow \mathbb{R}, \quad \text { with } \quad \alpha_{J}(0,0,0)=0,
$$

such that, if we set $\alpha=\alpha(\epsilon, \bar{\alpha}, \underline{\alpha}) \equiv\left(\bar{\alpha}, \underline{\alpha}, \alpha_{J}(\epsilon, \bar{\alpha}, \underline{\alpha})\right)$, then

$$
\mathcal{F}_{\epsilon, \alpha}^{N}[Z+W(\epsilon, \alpha)]=0
$$

for all $(\epsilon, \bar{\alpha}, \underline{\alpha}) \in B_{\delta}$. 
Proof. The theorem follows by the argument of Section 4 because that argument depends only the properties of $W$ identified in Definition 12, and on the structure of the restriction of the operator $\mathcal{M}-I$ to the Fourier 1-mode, where it agrees with its $N$-Fourier cutoff.

As a direct consequence of Theorem 27 we have the $N$-Fourier version of Theorem 28, from which Theorem 23 is evident:

THEOREM 28. Assume that $\Theta$ is nonresonant and let $W$ denote the solution (130) given by Corollary 26. Then there are constant $\delta>0$ and smooth functions $\alpha_{J}(\epsilon, \bar{\alpha}, \underline{\alpha})$ and $V_{\epsilon, \bar{\alpha}, \underline{\alpha}}$,

$$
\alpha_{J}=\alpha_{J}(\epsilon, \bar{\alpha}, \underline{\alpha}): B_{\delta} \rightarrow \mathbb{R} \quad \text { and } \quad V=V_{\epsilon, \bar{\alpha}, \underline{\alpha}}:: B_{\delta} \rightarrow \Delta^{N}
$$

such that

$$
V_{0,0,0}=Z \in \mathcal{K}, \quad \text { and } \quad \alpha_{J}(0,0,0)=0,
$$

and such that, if $\alpha$ is given by (90), then

$$
\begin{gathered}
\mathcal{F}_{\epsilon, \alpha}^{N}\left[V_{\epsilon, \bar{\alpha}, \underline{\alpha}}\right]=0, \quad \text { that is } \\
\mathcal{N}_{\alpha}^{N}\left[\mathbf{1}+\epsilon V_{\epsilon, \bar{\alpha}, \underline{\alpha}}\right]=\mathbf{1}+\epsilon V_{\epsilon, \bar{\alpha}, \underline{\alpha}},
\end{gathered}
$$

for all $(\epsilon, \bar{\alpha}, \underline{\alpha}) \in B_{\delta}$. Here the data for the nonlinear eigenvalue problem is

$$
V_{\epsilon, \bar{\alpha}, \underline{\alpha}} \equiv Z+W_{\epsilon, \alpha}
$$

where $Z=\mathcal{T}_{1}^{*} \mathbf{q}$ and $W_{\epsilon, \alpha}$ solves (130).

6. Conclusion. In this paper we have used a Liapunov-Schmidt decomposition to reduce the problem of existence of time-periodic solutions of the compressible Euler equations to the problem of finding solutions of the auxiliary equation (86) for $\epsilon>0$. The auxiliary equation is a perturbation of a linear operator that is invertible but whose inverse is unbounded due to the presence of small divisors. This introduces a new KAM type small divisor problem in bifurcation theory, for an operator which is a composition of linear jump operators and quasilinear evolution operators, the evolution being by the non-dimensionalized $p$-system. Although proving existence for this implicit function theorem appears to be beyond current mathematical techniques due to the quasilinear nature of the problem, analogous results are available for semilinear problems, and we conjecture that the problem does have a solution. To support this, we prove that the reduction goes through rigorously assuming an arbitrarily large Fourier cutoff. Given the many successes of KAM theory in resolving small divisor problems, one has to expect that these technical issues can be overcome to complete the proof that solutions with the periodic structure derived in [8] solve the compressible Euler equations exactly.

\section{REFERENCES}

[1] J. BourgaIN AND W. WANG, Quasi-periodic solutions of nonlinear lattice Schrodinger equations with random potential, J. Euro. Math. Soc., 10 (2008), pp. 1-45.

[2] W. CRAig AND G. WAYNE, Newton's method and periodic solutions of nonlinear wave equations, Comm. Pure Appl. Math., 46 (1993), pp. 1409-1498.

[3] K. Deimling, Nonlinear functional analysis, Springer, 1985. 
[4] M. Golubitsky and D.G. SChaeffer, Singularities and groups in bifurcation theory, SpringerVerlag, 1985.

[5] A. MAJDA, Compressible fluid flow and systems of conservation laws in several space variables, Applied Mathematical Sciences, no. 53, Springer-Verlag, 1984.

[6] P. H. Rabinowitz, Periodic solutions of nonlinear hyperbolic partial differential equations, Comm. Pure Appl. Math., 20 (1967), pp. 145-205.

[7] J. SMOlLer, Shock waves and reaction-diffusion equations, Springer-Verlag, New York, 1982.

[8] B. Temple And R. Young, A paradigm for time-periodic sound wave propagation in the compressible Euler equations, Methods and Appls of Analysis (2009), To appear, 26 pages, available at http://www.math.ntnu.no/conservation/2008/033.html.

[9] _ Time-periodic linearized solutions of the compressible Euler equations and a problem of small divisors, SIAM Journal of Math Anal (2009), To appear, 54 pages, available at http://www.math.ntnu.no/conservation/2008/034.html.

[10] R. Young, Global wave interactions in isentropic gas dynamics, Submitted, 47 pages, available at http://www.math.ntnu.no/conservation/2008/032.html, 2008.

[11] E. ZeHnder, Generalized implicit function theorems with applications to some small divisor problems, Comm. Pure Appl. Math., 28 (1975), pp. 91-140. 
B. TEMPLE AND R. YOUNG 